Review

\title{
Current Approaches, Challenges and Future Directions for Monitoring Treatment Response in Prostate Cancer
} \author{
Y.G. Man $1,6,8$ \\ 1. Bon Secours Cancer Institute, Bon Secours Health Care System, Richmond VA, USA. \\ 2. Division of Radiation Oncology, Bon Secours Health Care System, Richmond VA, USA. \\ 3. Virginia Urology, Richmond VA, USA. \\ 4. Department of Urology, Virginia Commonwealth University Health System, Richmond VA, USA \\ 5. Department of Radiology, Virginia Commonwealth University Health System, Richmond VA, USA. \\ 6. Division of Surgical Oncology, Bon Secours Health Care System, Richmond VA, USA \\ 7. INCORE, International Consortium of Research Excellence of the Theodor-Billroth-Adademy \\ 8. South Hospital of Nanjing, Nanjing, China.
}

T.J. Wallace 1,2,3凶 , T. Torre 1,2,3, M. Grob 4 , J. Yu 5, I. Avital 1,6 , BLDM Brücher 1,6,7, A. Stojadinovic 1,6,7 and

$\triangle$ Corresponding author: Timothy J. Wallace, MD, PhD. Division of Radiation Oncology, Bon Secours Cancer Institute (BSCI), Richmond, VA 23230 USA. Phone: (804) 266-7762. Fax: (804) 266-7994. E-mail: timothywallace1@gmail.com

() Ivyspring International Publisher. This is an open-access article distributed under the terms of the Creative Commons License (http://creativecommons.org/ licenses/by-nc-nd/3.0/). Reproduction is permitted for personal, noncommercial use, provided that the article is in whole, unmodified, and properly cited.

Received: 2013.09.19; Accepted: 2013.11.01; Published: 2014.01.01

\begin{abstract}
Prostate cancer is the most commonly diagnosed non-cutaneous neoplasm in men in the United States and the second leading cause of cancer mortality. One in 7 men will be diagnosed with prostate cancer during their lifetime. As a result, monitoring treatment response is of vital importance. The cornerstone of current approaches in monitoring treatment response remains the prostate-specific antigen (PSA). However, with the limitations of PSA come challenges in our ability to monitor treatment success. Defining PSA response is different depending on the individual treatment rendered potentially making it difficult for those not trained in urologic oncology to understand. Furthermore, standard treatment response criteria do not apply to prostate cancer further complicating the issue of treatment response. Historically, prostate cancer has been difficult to image and no single modality has been consistently relied upon to measure treatment response. However, with newer imaging modalities and advances in our understanding and utilization of specific biomarkers, the future for monitoring treatment response in prostate cancer looks bright.
\end{abstract}

Key words: prostate cancer, monitoring treatment response

\section{Introduction}

It is estimated that 240,000 men will be diagnosed with prostate cancer in 2013 making it the most commonly diagnosed non-cutaneous neoplasm in men in the U.S. Between 2006 and 2010, the median age at diagnosis of prostate cancer was 66 years. The age-adjusted incidence rate is 152.0 per 100,000 men per year. African American men have the highest incidence of disease at 228.5 per 100,000 men per year. Approximately $16 \%$ of men born today will be diagnosed with this disease in their lifetime and approx- imately 30,000 men will die from prostate cancer this year making it the second leading cause of cancer mortality in the U.S [1]. The importance of treating this disease and monitoring the success of these treatments cannot be overstated with regard to the impact it has on the healthcare system. There are many effective treatment options in the definitive treatment of prostate cancer.

Prior to the PSA-era, the effectiveness of treatment was judged by a lack of clinical progression or a 
resolution of symptoms. With the advent of PSA in the 1980s, monitoring of therapeutic success after completion of primary treatment for prostate cancer, while imperfect, was vastly improved over monitoring of clinical signs and symptoms as was historically solely relied upon. For all of the recent controversy surrounding PSA as a screening tool leading to sweeping changes in the recommendations for its use for the purpose of early detection, PSA remains the standard of practice in monitoring the state of disease in the post-treatment setting. Furthermore, as imaging techniques improve, more effective monitoring of disease response on the basis of anatomical and functional indicators is becoming possible. The purpose of this manuscript is to examine the current approaches, challenges and the future of monitoring treatment responses for patients with prostate cancer.

\section{Current Standard Treatment Options for Prostate Cancer}

Treatment for prostate cancer, like all cancers, is optimized according to stage of disease. Table 1 describes the American Joint Committee on Cancer Tumor-Node-Metastasis (AJCC TNM) staging system for prostate cancer. While TNM staging is important, it is not well suited for deciding the best treatment for a patient with prostate cancer. The reason for this limitation is the considerable heterogeneity of prognosis within each stage category. The National Comprehensive Cancer Network (NCCN) has stratified patients with prostate cancer into prognostic risk groups based on the pre-treatment serum PSA, biopsy Gleason score and clinical stage [2].

Table I. TNM Staging For Prostate Cancer

\begin{tabular}{|c|c|c|c|c|}
\hline Tx & \multicolumn{4}{|c|}{ cannot evaluate the primary tumor } \\
\hline T1 & \multicolumn{4}{|c|}{ tumor present, but not detectable clinically or with imaging } \\
\hline & T1a tumor $n$ & \multicolumn{3}{|c|}{ tumor was incidentally found in less than $5 \%$ of prostate tissue resected (for other reasons) } \\
\hline & tumor $\mathrm{n}$ & \multicolumn{3}{|c|}{ tumor was incidentally found in greater than $5 \%$ of prostate tissue resected } \\
\hline & T1c tumor $n$ & \multicolumn{3}{|c|}{ tumor was found in a needle biopsy performed due to an elevated serum PSA } \\
\hline \multirow[t]{4}{*}{ T2 } & \multicolumn{4}{|c|}{ the tumor can be felt (palpated) on examination, but has not spread outside the prostate } \\
\hline & T2a the tum & \multicolumn{3}{|c|}{ the tumor is in half or less than half of one of the prostate gland's two lobes } \\
\hline & the tum & \multicolumn{3}{|c|}{ the tumor is in more than half of one lobe, but not both } \\
\hline & T2c the tum & \multicolumn{3}{|c|}{ the tumor is in both lobes but within the prostatic capsule } \\
\hline \multirow[t]{3}{*}{ T3 } & \multicolumn{4}{|c|}{ the tumor has spread through the prostatic capsule (if it is only part-way through, it is still T2) } \\
\hline & T3a the tum & \multicolumn{3}{|c|}{ the tumor has spread through the capsule on one or both sides } \\
\hline & the tum & \multicolumn{3}{|c|}{ the tumor has invaded one or both seminal vesicles } \\
\hline T4 & \multicolumn{4}{|c|}{$\begin{array}{l}\text { tumor is fixed or invades adjacent structures other than seminal vesicles: such as external sphincter, rectum, bladder, levator } \\
\text { muscles, and/or pelvic side wall. }\end{array}$} \\
\hline $\mathbf{N x}$ & \multicolumn{4}{|c|}{ cannot evaluate the regional lymph nodes } \\
\hline NO & \multicolumn{4}{|c|}{ there has been no spread to the regional lymph nodes } \\
\hline N1 & \multicolumn{4}{|c|}{ there has been spread to the regional lymph nodes } \\
\hline Mx & \multicolumn{4}{|c|}{ cannot evaluate distant metastasis } \\
\hline M0 & \multicolumn{4}{|c|}{ there is no distant metastasis } \\
\hline \multirow[t]{4}{*}{ M1 } & \multicolumn{4}{|c|}{ there is distant metastasis } \\
\hline & the canc & \multicolumn{3}{|c|}{ the cancer has spread to lymph nodes beyond the regional ones } \\
\hline & the canc & \multicolumn{3}{|c|}{ the cancer has spread to bone } \\
\hline & the canc & \multicolumn{3}{|c|}{ the cancer has spread to other sites (regardless of bone involvement) } \\
\hline Gx & \multicolumn{4}{|c|}{ cannot assess grade } \\
\hline G1 & \multicolumn{4}{|c|}{ the tumor closely resembles normal tissue (Gleason 2-4) } \\
\hline G2 & \multicolumn{4}{|c|}{ the tumor somewhat resembles normal tissue (Gleason 5-6) } \\
\hline G3-4 & \multicolumn{4}{|c|}{ the tumor resembles normal tissue barely or not at all (Gleason 7-10) } \\
\hline Stage & Tumor & Nodes & Metastases & Grade \\
\hline Stage I & T1a & N0 & M0 & G1 \\
\hline Stage II & T1a & No & M0 & G 2-4 \\
\hline & $\mathrm{T} 1 \mathrm{~b}$ & N0 & M0 & Any G \\
\hline & T1c & No & M0 & Any G \\
\hline & $\mathrm{T} 1$ & No & M0 & Any G \\
\hline & $\mathrm{T} 2$ & N0 & M0 & Any G \\
\hline Stage III & T3 & No & M0 & Any G \\
\hline Stage IV & $\mathrm{T} 4$ & N0 & M0 & Any G \\
\hline & Any $\mathrm{T}$ & N1 & M0 & Any G \\
\hline
\end{tabular}


Table 2 shows the stratification of the groups according to risk: very low risk, low risk, intermediate risk, high risk and very high risk disease. The very low risk group includes T1c disease with Gleason score $\leq 6$, PSA $<10,<3$ positive biopsy cores and $\leq$ $50 \%$ in any core and a PSA density $<0.15 \mathrm{ng} / \mathrm{ml} / \mathrm{g}$. The low risk group includes stage T1-T2a disease and Gleason score $\leq 6$ and a PSA $<10$. The intermediate risk group includes stage T2b/T2c disease or Gleason score 7 or PSA 10 to 20 . The high risk group includes stage T3a or Gleason score 8 to 10 or PSA > 20; and, the very high risk group includes those patients with $\mathrm{T} 3 \mathrm{~b}$ or T4 disease. The treatment options for the risk stratified subgroups may consist of active surveillance, surgery (both radical prostatectomy and robotic assisted laparoscopic prostatectomy), brachytherapy, external beam radiation therapy (photon IMRT, protons, cyberknife etc.), androgen deprivation therapy, chemotherapy or a combination of 2 or more of the above modalities. The treatment decision depends on the patient's risk group assigned at diagnosis, the patient's projected survival and the patient-specific preferences.

In addition to the NCCN guidelines, other sources offer "best-practice" advice and guidelines to aid health care providers and their patients in making the most informed decisions regarding the treatment options for this disease. The guidelines outlined by the National Guideline Clearinghouse (NGC), which is an initiative of the agency for Healthcare Research and Quality (AHRQ), U.S. Department of Health and Human Services, were designed to provide an accessible mechanism for obtaining objective, detailed information on clinical practice guidelines for the treatment of prostate cancer and to further their dissemination, implementation and clinical use [3]. In 2008, The National Institute for Health and Clinical Excellence (NICE) released guidelines and recommendations regarding the treatment and care of men with prostate cancer in the National Health Service (NHS) in England and Wales [4]. Both the American Urological Society and the European Association of Urology (EUA) offer detailed guidelines for the diagnosis and treatment of men with prostate cancer $[5,6]$. While there are subtle differences between the guidelines, treatment options outlined in each are fairly consistent and stratified by risk/stage classification. Table 3 lists standard treatment options based on tumor stage recommended by all mentioned guidelines.

Table 2. Risk stratification for men with localized prostate cancer.

\begin{tabular}{|c|c|c|c|c|c|}
\hline Risk Stratification & PSA & Gleason Score & & Clinical Stage & \\
\hline Very Low Risk & $\begin{array}{l}<10 \mathrm{ng} / \mathrm{ml} \\
\text { (and PSA density }<0.15 \mathrm{ng} / \mathrm{ml} / \mathrm{g} \text { ) }\end{array}$ & and & $\begin{array}{l}\leq 6 \\
\text { (and }<3 \text { positive cores and } \\
\leq 50 \% \text { of any one core) }\end{array}$ & and & T1-T2a \\
\hline Low Risk & $<10 \mathrm{ng} / \mathrm{ml}$ & and & $\leq 6$ & and & T1-T2a \\
\hline Intermediate Risk & $10-20 \mathrm{ng} / \mathrm{ml}$ & or & 7 & or & $\mathrm{T} 2 \mathrm{~b}-\mathrm{T} 2 \mathrm{c}$ \\
\hline High Risk & $>20 \mathrm{ng} / \mathrm{ml}$ & or & $8-10$ & or & $\mathrm{T} 3 \mathrm{a}$ \\
\hline Very High Risk & & & & & $\mathrm{T} 3 \mathrm{~b}$ or $\mathrm{T} 4$ \\
\hline
\end{tabular}

Table 3. Standard treatment options based on tumor stage recommended by all guidelines.

\begin{tabular}{ll}
\hline TNM Staging & Standard Treatment Options \\
\hline Stage I & Watchful waiting or active surveillance \\
& Radical prostatectomy \\
& External-beam radiation therapy (EBRT) \\
& Interstitial implantation of radioisotopes \\
& Watchful waiting or active surveillance \\
& Radical prostatectomy \\
Stage II & External-beam radiation therapy (EBRT) with or without hormonal therapy \\
& Interstitial implantation of radioisotopes \\
& External-beam radiation therapy (EBRT) with or without hormonal therapy \\
& Hormonal manipulations (orchiectomy or luteinizing hormone-releasing hormone [LH-RH] agonist) \\
Stage III & Radical prostatectomy with or without EBRT \\
& Watchful waiting or active surveillance \\
& Hormonal manipulations \\
& Bisphosphonates \\
Stage IV & External-beam radiation therapy (EBRT) with or without hormonal therapy \\
& Palliative radiation therapy \\
& Palliative surgery with transurethral resection of the prostate (TURP) \\
& Watchful waiting or active surveillance \\
& Chemotherapy for hormonal management of prostate cancer \\
& Imunotherapy \\
\hline
\end{tabular}




\section{Very low-risk disease}

Very low risk disease is defined as follows: Serum PSA $<10 \mathrm{ng} / \mathrm{ml}$ (and PSA density $<0.15$ $\mathrm{ng} / \mathrm{ml} / \mathrm{g}$ ) and Gleason Score $\leq 6$ (and $<3$ positive cores and $\leq 50 \%$ of any one core) and Clinical Stage T1-T2a. The main goal in a patient with very low risk disease is active surveillance if the life expectancy is estimated to be $<20$ years. When a patient with a life expectancy of $<10$ years presents with all of the very low risk disease characteristics listed in Table 2, Serum PSA should be checked at least as often as every six months and a digital rectal examination (DRE) should be performed at least as often as every 12 months. When a patient with very low risk disease presents with a life expectancy of $>10$ years but $<20$ years a serum PSA should be performed at least as often as every six months and a DRE performed as often as every 12 months. A repeat prostate biopsy should also be performed at least as often as every 12 months. If the life expectancy is $\geq 20$ years on presentation with very low risk prostate cancer then the goal of treatment is cure and the patient is treated according to the low risk algorithm below.

\section{Low-risk disease}

Low risk disease is defined by the following parameters: PSA $<10 \mathrm{ng} / \mathrm{ml}$ and Gleason Score $\leq 6$ and Clinical Stage T1-T2a. The main goal of treatment in a patient with low-risk disease is cure. When a patient presents with low-risk disease, as specified in Table 2, and has a projected life expectancy estimated to be < 10 years the treatment choices include active surveillance, external beam radiation therapy or brachytherapy. If the life expectancy on presentation of low-risk disease is estimated to be $\geq 10$ years the treatment options include those above for patients with a life expectancy of $<10$ years, but also includes the additional treatment option of radical prostatectomy +/- lymph node dissection depending on the patient's suitability for surgery (operability) and personal treatment preference.

\section{Intermediate-risk disease}

Intermediate risk disease is defined by the following parameters: PSA of $10-20 \mathrm{ng} / \mathrm{ml}$ or Gleason Score $=7$ or Clinical Stage T2b-T2c. The main goal of treatment for a patient diagnosed with intermediate-risk prostate cancer is cure. When a patient presents with intermediate-risk disease as outlined in Table 2 and has an estimated life expectancy of $<10$ years, the treatment options include external beam radiation therapy +/- androgen deprivation, with or without an interstitial brachytherapy boost or expectant management. If the life expectancy of the pa- tient presenting with intermediate-risk disease is $\geq 10$ years radical prostatectomy +/- lymph node dissection is an excellent option depending on the patient's preference and suitability for surgery. There is debate about the preferred management of this disease as few randomized trials have compared effectiveness between primary treatments. Treatment choice may also be influenced by the functional impact of treatment including potential loss of erectile dysfunction, urinary incontinence or other side effects from treatments, which have been documented in the literature [7].

\section{High-risk disease}

The following parameters define high risk prostate cancer: PSA $\geq 20 \mathrm{ng} / \mathrm{ml}$ or Gleason Score 8-10 or Clinical Stage T3a. The main goal of treatment in a patient diagnosed with high-risk prostate cancer is cure. Radiation therapy given in combination with androgen deprivation for 2-3 years has shown to significantly improve disease-free survival [8-10]. Radiation therapy should include the pelvic lymph nodes if their risk of involvement exceeds 15\% [11]. Radical prostatectomy plus pelvic lymph node dissection may be considered in highly selected patients with low-volume disease without fixation to the pelvic musculature or skeleton [2]. The use of systemic chemotherapy in combination with radiation therapy and androgen suppression is under investigation, but currently plays no role in locally advanced high-risk prostate cancer. Androgen deprivation therapy alone may be used as a stabilizing agent to delay disease progression in a patient with locally advanced high-risk disease whose life expectancy is estimated to be $<10$ years. If a patient is not a good surgical candidate or if he is not a good candidate for radiation therapy (including inflammatory bowel disease or prior radiation therapy), isolated androgen deprivation therapy may be used.

\section{Very high-risk disease}

Very high risk disease is defined as clinical stage T3b to T4 (locally advanced) disease. The treatment options for patients with very high-risk disease are the same as those for patients with high-risk disease, noted above.

\section{Metastatic disease}

The main goal of the treatment for metastatic prostate cancer is palliation of symptoms. The primary treatment of choice for metastatic prostate cancer is androgen deprivation with an LHRH agonist or 
antagonist. Patients will usually receive a nonsteroidal anti-androgen (e.g., bicalutamide) for a few days prior to and for a week following the initiation of LHRH agonist. It has been shown that the addition of a first-generation bisphosphonate improves overall survival in men with metastatic prostate cancer [12]. It is also recommended that an intravenous bisphosphonate (e.g., zoledronic acid) be given in the presence of castrate-resistant metastatic disease to reduce the number of skeletal-related events [13]. A randomized trial showed that toremifene, a selective estrogen receptor modulator, significantly decreased the incidence of new vertebral fractures in men receiving androgen deprivation therapy for metastatic prostate cancer [14]. It also significantly improved bone mineral density, bone turnover markers and serum lipid profiles. A newer agent, Denosumab (Xgeva) which is a human monoclonal antibody that inhibits RANK ligand, has been found to be superior to the bisphosphonate zoledronic acid in preventing skeletal-related events in patients with advanced prostate cancer and bone metastases [15].

Systemic chemotherapy should be reserved for castrate-resistant metastatic disease. Docetaxel chemotherapy is the current standard of care for metastatic castrate-resistant prostate cancer [16,17]. In patients with metastatic castrate-resistant prostate cancer experiencing progression after initial chemotherapy with a docetaxel-based regimen, oral satraplatin has been shown to delay progression of disease as well as disease-related pain [18]. Alternative regimens for disease nonresponsive to preferred chemotherapy include abiraterone, mitoxantrone or carbazitaxel [2]. Sipuleucel $-\mathrm{T}$ is recommended for asymptomatic or minimally symptomatic patients with castrate-resistant metastatic disease and an ECOG performance status of 0-1. It is not recommended for patients with visceral disease and a life expectancy of $<6$ months [19].

External beam radiation therapy in palliative doses can be given to sites of painful bony metastases. Radiation may include a single fraction or a more protracted 1- or 2- week course depending on normal tissue toxicity and patient convenience. Systemic radiation therapy (e.g., strontium-90, samarium-153) has been used in the past to relieve pain from bony metastases [20]. Recently Radium-223 has been FDA-approved for use in the treatment of metastatic castrate-resistant prostate cancer. It is a calcium mimetic that localizes to bone and delivers radiation directly to bone metastases by emitting an alpha particle. It has shown efficacy at both preventing skeletal-related events and prolonging overall survival [21].

\section{Treatment Options under Clinical Evaluation for Locally Advanced Prostate Cancer Cryotherapy}

Cryotherapy is a technique whereby, under ultrasound guidance, special metal rods are inserted through the perineum into the prostate. Highly purified argon gas is used to cool the tips of the rods and freeze the surrounding tissue, thereby killing the prostate cancer. A catheter filled with warm saline prevents the urethra from freezing during cryotherapy. Impotence occurs in up to $90 \%$ of cases. The method has been studied as a salvage therapy after primary treatment failure [22], but also is now being studied in the primary treatment of organ-confined prostate cancer [23]. The poor quality of available studies makes it difficult to determine the relative benefits of this modality. Randomized trials are needed to fully evaluate the potential of cryotherapy in patients with prostate cancer [24].

\section{High-intensity focused ultrasound (HIFU)}

In HIFU therapy, high-intensity ultrasound beams are precisely focused on diseased tissue, raising the temperature within the target tissue to between $65^{\circ} \mathrm{C}$ and $85^{\circ} \mathrm{C}$, destroying the diseased tissue by coagulation necrosis. This treatment is currently being studied in the United States. There have been a number of studies in Asia showing a potential benefit to HIFU in localized prostate cancer, but this is not currently an accepted standard of practice [25-28].

\section{Focal Therapy}

Focal treatment in prostate cancer has been shown to be a potential treatment of recurrent disease [29-31]. Definitive focal therapy is an emerging treatment modality for localized prostate cancer that aims to reduce the morbidity seen with radical therapy, while maintaining cancer control. Focal therapy treatment strategies attempt to minimize damage to normal tissue, especially anatomic structures that are important functional determinants: the neurovascular bundles, external sphincter, bladder neck and rectum. There are a number of ablative technologies that can deliver energy to destroy cancer cells as part of a focal therapy strategy. The most widely investigated are cryotherapy and high-intensity focused ultrasound, as mentioned above $[30,31]$. Existing radical therapies, such as brachytherapy and external beam radiotherapy, also have the potential to be applied in a focal manner [29]. Recent Phase I/II data has been promising indicating a potential role for definitive focal therapy [32]. The Index Study was proposed this year and represents the first prospective multicenter trial 
to evaluate the outcomes of a tissue preserving strategy for localized prostate cancer [33].

\section{Monitoring Treatment Response in Pros- tate Cancer}

\section{Overview of Current Standard Approaches to Monitoring treatment Response}

It is important to monitor patients after definitive treatment for prostate cancer in order to identify locally recurrent disease at a point where further curative treatments may be employed. Equally important is the need to identify and treat complications of the initial therapy. The cornerstone of follow-up in monitoring for treatment response after definitive treatment for prostate cancer is the PSA. In one study it was shown that $45 \%$ of patients experienced recurrence in the first two years, $77 \%$ in the first five years and $96 \%$ by ten years following definitive therapy [34]. It has also been shown that a serum PSA recurrence nearly always precedes a clinical recurrence [35]. Therefore, the current standard for monitoring includes serum PSA every 6 to 12 months for the first five years of post-treatment surveillance and then annually after that $[2,6]$. PSA testing every three months may be recommended for men at high risk of recurrence. Because, on rare occasions, a local recurrence may occur in the absence of an elevated PSA, digital rectal examinations should be done annually $[2,6]$. Any new nodule felt on physical exam should raise suspicion of recurrent disease.

Prostate cancer is unique among cancers in that it usually has no associated symptoms and is rarely measured radiographically. The biomarker, PSA, is a "leading indicator" in prostate cancer detection and is often the first and only sign that cancer is present. The use of PSA to monitor treatment response, although not perfect, has for years been the gold standard by which various curative approaches are compared.

Transrectal Ultrasound (TRUS) and biopsy are not a part of the recommended routine monitoring after treatment for prostate cancer. Ultrasound alone is never used in the absence of a biopsy and the combination is only used to obtain pathologic confirmation of a local recurrence identified by serum PSA and/or DRE.

A bone scan is not routinely used in monitoring treatment response in asymptomatic patients. It is, however used to further evaluate a rising serum PSA in the absence of symptoms or to evaluate new symptoms in the presence or absence of a detectable PSA. Furthermore computed tomography (CT) and magnetic resonance imaging (MRI) currently have no well-defined role in monitoring for routine treatment response after definitive treatment in the absence of symptoms or a detectable PSA. Multiparametric prostate MRI is, however gaining increased acceptance for the assessment of response to treatment and will be discussed later in this manuscript. A CT scan may be used as a secondary staging study to further evaluate for potential salvage treatments after a diagnosis of recurrence has been made.

Current approaches and challenges, in addition to the potential future directions, for monitoring treatment responses will differ slightly depending on which specific therapeutic modality was used in the initial phase of treatment of the disease and will be looked at separately.

\section{Current State of Objective Response Criteria in Prostate Cancer}

Objective response criteria in oncology are important as they represent the standard by which the efficacy of therapeutic agents is determined in cancer trials. Bone metastases are a common manifestation of advanced prostate cancer with an incidence of up to $70 \%$ in autopsy studies [36]. Measuring response to treatment of prostate cancer can be both subjective and objective. The subjective relief of pain, neurologic deficits or obstructive symptoms can be more important to the patient than an objective response to treatment however, measuring an objective tumor response is important when measuring and comparing effectiveness of treatments. The first generation of these response criteria was the International Union Against Cancer (UICC) published in 1977 [37] and the World Health Organization (WHO) published in 1979 [38]. Since those criteria were published, the quality and diagnostic accuracy of imaging modalities have improved tremendously and have been replaced with more up-to-date response criteria. However, none of them, even today, apply to prostate cancer, as they remain inefficient.

The most commonly used set of criteria to measure response to treatment is the Response Evaluation Criteria in Solid Tumors (RECIST) originally published in 2000 [39]. These criteria focus predominantly on the physical measurement of solid tumors. The response criteria used to determine objective tumor response include complete response which is the disappearance of all target lesions, partial response which is at least a $30 \%$ decrease in the sum of diameters of target lesions, progressive disease which is defined as a $20 \%$ or greater increase in the sum of diameters of target lesions; and, stable disease, which is defined by insufficient shrinkage or increase in target lesion dimensions. In the RECIST criteria, bone metastases are considered too difficult to measure objectively and are designated as unmeasurable [39]. The updated RECIST 1.1 criteria include bone metastases 
as target lesions when they have associated soft tissue components measuring $>/=10 \mathrm{~mm}[40]$. Therefore the majority of bone metastases from prostate cancer are still considered unmeasurable according to these updated criteria and their response to treatment cannot be measured quantitatively. However, should the imaged bone metastases increase $20 \%$ in the sum of the greatest tumor diameters, they may contribute to the classification of overall patient response through the designation, 'unequivocal progression' [40].

Other criteria have been developed that consider the difficulty in evaluating bone metastases objectively. In 2004, the University of Texas, MD Anderson Cancer Center developed a bone-specific set of response criteria (MDA Criteria) that incorporated both CT and MRI, and include both quantitative and qualitative assessments of the behavior of bone metastases [41]. In one study retrospectively comparing the MDA criteria with the WHO criteria, the MDA criteria were shown to better differentiate responders from nonresponders [42]. A recently published prospective comparison showed that both the WHO criteria and MDA criteria predicted progression free survival (PFS) at six months based on the binary classification of progressive disease or non-progressive disease, but these rates, as well as overall survival did not differ at 3 months [43].

The next generation of response criteria emerged in 2009, with the publication of the PERCIST criteria. Taking advantage of the functional measurement of FDG-PET and the increased glucose metabolism in most solid tumors, an effort was made to publish updated objective response criteria aimed at standardizing with increasing accuracy functional response of metastatic lesions to treatment [44]. The addition of "functional measurement" allowed the percentage of change in metabolic activity from baseline and the number of weeks since the initiation of therapy to be recorded to provide a continuous plot of tumor activity and treatment response. These functional measurements were not adopted by the RECIST working group in their revisions because of the difficulty in standardizing PET/CT evaluations [40].

\section{Challenges in the application of Objective Re- sponse Criteria in Prostate Cancer}

In one publication, patients enrolled on institutional review board-approved trials were assessed [45]. In addition to the application of RECIST-defined outcome measures for tumor regression in the metastatic patient cohorts, the RECIST criteria were also applied to the states of a rising PSA and localized disease as well. When using the RECIST criteria only $43.5 \%$ of men with castrate metastatic and $16 \%$ of noncastrate metastatic disease had measurable le- sions. More significant was the fact that there were no target lesions defined in patients with a rising PSA and localized disease. In fact, the PSA based eligibility and outcomes under RECIST conflict with the accepted standards of reporting rising PSA in the post-treatment state. According to the RECIST criteria, tumor marker values are recorded as "normal" or "abnormal" $[39,40]$, when in reality, monitoring PSA after treatment for any stage of prostate cancer currently occurs across a broad spectrum of values with varying definitions of recurrence based on which initial treatment modality was pursued.

Another challenge in the application of the RECIST criteria to prostate cancer patients is that the presence or absence of symptoms is not considered an eligibility criterion [41]. While overall survival is usually the desired endpoint in most studies, the palliation of pain [46] and the reduction in the risk of skeletal metastases [47] have also been used to measure the success of treatment regimens in prostate cancer.

\section{Future Application of Objective Response Criteria in Prostate Cancer}

Whereas most solid tumors may be assessed reliably with the RECIST criteria, prostate cancer cannot. The inability to include the treatment response of an "unmeasurable' bone metastasis or a detectable PSA after a prostatectomy underscores this challenge and makes it difficult to accurately and consistently monitor disease in the post-treatment setting. With the increased number of treatment options for prostate cancer, both localized and castrate-resistant disease, one must be able to measure success of treatment according to the treatment objective for the individual agent and in the individual patient to whom it is offered. While a "one shoe fits all" approach may be appropriate for some solid tumors, monitoring treatment response in prostate cancer must be tailored to the individual management scenario, whether that initial modality is active surveillance, surgery, radiation or treatment for metastatic disease.

\section{Current Approaches in Monitoring Disease During Active Surveillance}

In 2010, the NCCN guidelines introduced a new risk stratification category called "very low risk" that incorporated the most stringent disease criteria to include clinical stage T1-T2a disease with a PSA < $10 \mathrm{ng} / \mathrm{ml}$ ( with a PSA velocity of $<0.15 \mathrm{ng} / \mathrm{ml} / \mathrm{g}$ ) and $<3$ cores positive with $<50 \%$ involvement of Gleason score $3+3$. Active surveillance is recommended as the sole initial approach for men who have a life expectancy of $>20$ years [2]. In the 2011 updated NCCN Guidelines, active surveillance monitoring was made more rigorous for men in the "very low risk" category 
and now includes a serum PSA every six months, a digital rectal (prostate) exam (DRE) should be performed as often as every 6 months but at least every 12 months and a repeat biopsy should be considered as often as every 12 months. Repeat biopsies are not indicated in men $>75$ years of age or in those with a life expectancy $<10$ years [2]. A recent cohort study published in 2010 showed, at a median follow-up of 6.8 years, a 10 -year overall survival of $68 \%$ in patients undergoing active surveillance [48]. At 10 years, the disease-specific survival was $97.2 \%$ with $62 \%$ of men still alive on active surveillance. Of the $30 \%$ of men who subsequently underwent definitive treatment $48 \%$ had a PSA doubling time of $<3$ years, $27 \%$ had a Gleason score progression on repeat biopsy and 10\% were treated as a result of patient preference.

\section{Challenges in Monitoring During Active Sur- veillance}

The biggest challenge in monitoring patients under active surveillance is, knowing when to make the decision to offer definitive treatment, and when to continue surveillance. Generally there are three reasons that a person will proceed with treatment: 1) Gleason score progression, 2) a rapidly rising PSA (increasing PSA velocity) or 3) patient anxiety. A Gleason score progression is less controversial and an increase on a subsequent biopsy to $>/=7$ would warrant consideration of definitive treatment.

PSA kinetics (PSA doubling time and PSA velocity) are less helpful when determining disease progression. Historically, a PSA doubling time with a cut-off value ranging from $\leq 2$ years to $\leq 4$ years was a criterion for active treatment [6]. However, in a study of 290 men who met the criteria for active surveillance, 35\% developed pathologic progression at a median follow up of 2.9 years. PSA doubling time was not significantly associated with subsequent adverse biopsy findings and PSA velocity was only marginally significant suggesting that PSA kinetics do not reliably predict disease biology [49]. Furthermore, a potential challenge in monitoring during active surveillance is a rising PSA in the presence of a consistently negative biopsy. There is growing evidence to suggest that the use of MRI may be beneficial in finding more "anteriorly" situated tumors that may not be readily sampled during a standard transrectal biopsy [50].

Patient anxiety has been shown to be a significant factor in the decision to proceed with definitive treatment, and might affect up to $10 \%$ of patients who undergo treatment for prostate cancer [51]. It has more recently been suggested that the psychosocial aspect or burden of living with prostate cancer plays a substantial role in adherence to active surveillance and outcomes of men with the disease [52]. Effective clinician education and counseling as well as early referral to supplemental support services should be implemented to alleviate these fears that may precipitate unnecessary treatment. Another challenge of active surveillance may be the discordance between a rising PSA and a continued negative biopsy.

Caution also needs to be taken when using guidelines for surveillance. The NICE guidelines were published based mainly on data from heavily screened patients in North America. It was shown recently that these criteria should not be used when counseling men in the United Kingdom with regard to active surveillance [53]. Seven hundred consecutive men treated for prostate cancer from 2005 by robot-assisted laparoscopic prostatectomy (RALP) were included. Patients satisfying NICE criteria for low-risk disease (PSA level $<10 \mathrm{ng} / \mathrm{mL}$ and Gleason score $\leq 6$ and stage cT1-2a) had their pathological samples analyzed for advanced disease, defined as extracapsular extension (ECE: pT3), seminal vesicle involvement (SVI), Gleason sum 7, or 8-10 or node-positive disease. In all, 275 patients $(39.2 \%)$ met the NICE low-risk criteria, but pathologically advanced disease was found in $37.2 \%$ of this group who would not have been considered for active surveillance.

\section{Current Approaches in Monitoring Treatment Response after Surgery}

Pathology

Several surgical options are available for removal of the prostate gland. Whether an open retropubic radical prostatectomy, a DaVinci robotic prostatectomy (or robot-assisted laparoscopic prostatectomy), or radical perineal prostatectomy is performed, the goal is the complete removal of the prostate gland and all cancerous cells. Pathologic analysis of prostatectomy specimens is the first tool used to measure surgical outcome and plays an important role in predicting 'biochemical-free survival'. Adverse prognostic indicators such as high Gleason scores, positive surgical margins, extracapsular extension, and seminal vesicle involvement are independent risk factors for recurrent disease and are the standard measures used to determine outcome and the need for adjuvant or salvage therapy [34,54-59]. Recurrence rates exceeding $50 \%$ in patients with these adverse pathologic features have been reported $[60,61]$. Three randomized trials to include SWOG 8794, EORTC 22911, and ARO 96-02 have documented significant improvements in biochemical recurrence-free survival when adjuvant radiotherapy following prostatectomy was compared with prostatectomy alone [60-63]. There now is enough evidence to support adjuvant radiotherapy for adverse pathologic findings to include seminal vesicle invasion, positive surgical margins 
and extraprostatic extension; as such, the new AUA/ASTRO guidelines recommend informing patients of the benefits and offering adjuvant radiotherapy [63].

Gleason score is another pathologic measure used following surgery. A Gleason score of 8 or greater has been associated with a higher incidence of biochemical relapse and reduced prostate cancer specific survival [34]. This is not a surprise as high risk Gleason scores are one of the strongest indicators of metastatic disease. Micro-metastatic disease in this setting is considered to be the primary reason for a rising PSA.

Some controversy exists regarding the value of lymphovascular invasion (LVI) in assessing radical prostatectomy specimens, but most recent studies have found LVI to be important. Yee and colleagues from Memorial Sloan-Kettering Cancer Center have demonstrated the presence of LVI in approximately $10 \%$ of 1200 recent prostatectomy specimens. Clear evidence of tumor in endothelial spaces was required to designate a tumor specimen as having lymphovascular invasion (LVI). LVI was shown to correlate with other well-established predictors of biochemical relapse such as Gleason score, extracapsular extension and nodal involvement. In fact, $>50 \%$ of patients with positive nodes demonstrated LVI. In multi-variate analysis, the presence of LVI was minimally helpful; the authors suspected that follow-up was too short for an additional variable to contribute much to accuracy of estimating prognosis over that provided by standard pathologic features [64].

These findings are similar to those of Shariat et al who demonstrated LVI in 5\% of approximately 600 prostatectomy specimens. LVI was associated with extracapsular extension beyond the prostate, seminal vesicle involvement and positive surgical margins. On univariate analysis, LVI significantly correlated with biochemical relapse but was not found to be an independent prognostic factor for biochemical relapse on multivariate analysis. LVI was, however, associated with both metastases and death. These same researchers found that while peri-neural involvement was also associated with other worrisome pathological features, it was not independently prognostic for metastases or death [65].

May et al also found LVI in 10\% of prostatectomy specimens. Similar to other studies, LVI correlated with pre-operative PSA, Gleason score and seminal vesicle involvement. Unlike most other studies, however, LVI was also associated with biochemical relapse on multi-variate analysis [66].

Older studies did not show LVI to be an independent predictor of relapse, but in 2007, the Association of Directors of Anatomic and Surgical Pathology finally changed their recommendations to include reporting of LVI. Prior to that, LVI reporting was considered optional [67].

The presence of lymph node metastases at the time of radical prostatectomy has long been regarded as a poor prognostic sign. Early in the PSA era and prior to the PSA era, if a positive lymph node was identified at the beginning of a radical prostatectomy, the prostatectomy was often aborted. The disease was then treated without curative intent. The incidence of positive nodes has certainly declined in the PSA era. Some centers such as the Mayo clinic however, have taken an aggressive treatment approach to lymph node positive disease. Cheng et al were among the first to demonstrate that radical prostatectomy including bilateral pelvic lymphadenectomy plus orchiectomy was superior to orchiectomy alone. The early reports were not randomized trials and the level of evidence did not meet today's standards to broadly impact clinical practice [68]. Messing et al reported a prospective trial on behalf of the Eastern Cooperative Oncology Group that immediate androgen deprivation was superior to a delayed approach in node positive patients [69]. The initial study was criticized for lack of centralized review among other issues but the results were confirmed with follow-up analysis [70]. Despite these studies, there is no definite consensus on what is the best approach for lymph node positive disease.

\section{PSA}

In addition to pathologic review, serum PSA is obtained to measure surgical outcome. Within four to six weeks following surgery, it is expected that PSA should be "zero" based on the biomarker's half -life of only 2-3 days. Biochemical failure defined by the AUA is a PSA of $>0.2 \mathrm{ng} / \mathrm{mL}$ on two or more measures [71]. However, many clinicians now consider any consistent value $>0.1 \mathrm{ng} / \mathrm{mL}$ to be indicative of recurrence and worthy of consideration for salvage treatment. If PSA never falls to the undetectable level following surgery and/or is rapidly rising, the implication is that systemic disease or at least a component of systemic disease is present [72]. If the PSA rises slowly or if the rise occurs after being undetectable for two or more years then this predicts a local only recurrence within the prostatic bed [34,73].

Numerous retrospective studies have shown the benefits of salvage radiation therapy once biochemical recurrence after prostatectomy is present [74]. Defining the optimal PSA level to be used as the threshold for initiation of the therapy is much more complicated. There exists controversy over when a biochemical failure represents clinically significant disease that requires salvage treatments and if earlier therapy 
provides superior outcomes. King from UCLA performed a systematic review of over 60 published salvage radiation therapy studies of which 41 met the selection criteria looking at biochemical relapse as related to the PSA level at the start of radiation. He found that relapse free survival was compromised on an average $2.6 \%$ for each $0.1 \mathrm{ng} / \mathrm{mL}$ rise in the pretreatment PSA. The range of PSA was $0.25-3.7 \mathrm{ng} / \mathrm{mL}$. When he extrapolated, he found that when radiation was initiated with a PSA level of 0.2 or less, relapse free survival approached 64\% [75]. Stephenson et al. developed a nomogram looking at predictors for salvage radiation and found an improved biochemical response to treatment when the treatment was initiated prior to a PSA level of $1 \mathrm{ng} / \mathrm{mL}$ [76].

One study has shown a somewhat surprising association between PSA doubling time after radical prostatectomy and response to salvage irradiation. In a retrospective analysis of 635 men treated at Johns Hopkins medical center, patients with a PSA doubling time of $<6$ months actually showed a survival advantage compared to those with longer doubling times. While men with PSA doubling time $<6$ months did worse overall compared to their counterparts with longer doubling times, they seemed to receive the most benefit from salvage therapy. The implication is that men with longer doubling times may have biologically less aggressive disease [77].

A recent study by Karlin et al. made an interesting observation regarding PSA thresholds for salvage radiotherapy relative to Gleason score. After adjusting for margin status, their data suggest that patients with Gleason score 8 - 10 should be offered salvage treatment at the earliest detectable point while patients with Gleason score $\leq 7$ may have the opportunity to be followed [78].

Outcome measures after prostatectomy are multi-factorial and should include pathologic features, PSA parameters such as supersensitive PSA and PSA kinetics, and need to be considered collectively when used to predict for biochemical recurrence. These measures can help determine those candidates that are more likely to benefit from adjuvant or salvage therapies as well as help determine the best time to initiate the therapy in order to maximize patient outcomes.

\section{Challenges in Monitoring Treatment Response after Surgery}

There are many challenges of monitoring treatment response in prostate cancer, mainly which center around the use of PSA as the marker of disease. Whether prostate cancer is treated with surgery, radiation or other forms of therapy, PSA is really the only current tool that is readily available, standardized, and utilized in the community setting. All prostate cells produce PSA; however, not all prostate cancer cells produce PSA in a direct correlation with tumor volume. Poorly differentiated tumors may lack the expression of PSA; therefore it may not measure accurately the burden of disease [79].

Another problem with PSA as a leading indicator of disease is that the presence of PSA does not reveal the location of disease. Distinguishing between locally persistent disease vs. metastatic disease is a dilemma for accurate treatment decisions. Often patients are given the benefit of the doubt and offered salvage treatment with the hopes that the observed PSA elevation represents local disease. The practitioners are faced with trying to incorporate multiple variables such as pathologic factors, PSA kinetics, and patient factors including age, co-morbidities, and expected longevity.

Although PSA can potentially detect the presence of cancer cells, it can also detect the presence of normal prostate cells. Detectable PSA after radical prostatectomy is universally felt to be due to residual or recurrent prostate cancer. There is a small possibility that detectable but very low and stable PSA, may be due to retained benign tissue. There are reports of surgical margins with benign tissue as high as $58 \%$ in robotic assisted laparoscopic prostatectomies [80]. To date, there are no published reports that conclusively demonstrate a higher rate of PSA recurrence to be associated with specimens with benign glands at the margin. Godoy et al wrote that in a properly performed radical prostatectomy, measurable PSA attributed to retained benign prostatic tissue should be an extraordinarily rare event [81]. Still it may be prudent to have a period of close observation for patients with very low but detectable stable PSA in the range < $0.2 \mathrm{ng} / \mathrm{ml}$.

Other normal tissue cells may also produce PSA. PSA immunoreactivity has been found in some non-prostatic tissues in small amounts such as parotid gland, apocrine glands of the skin, bladder, ovary, pancreatic tissues and breast [82-85]. In fact, efforts have been made to investigate PSA utilization as a marker of breast cancer but with little success [85]. Diagnostic dilemmas have been reported in the setting of treated prostate cancer and the development of a parotid oncocytoma. Contribution to serum PSA from any other source other than prostate tissues should be an extremely rare event, but as PSA assays become more sensitive, it is prudent to keep in mind that PSA is not only produced by prostatic tissues [83]. 


\section{Current Approaches in Monitoring Treatment Response after Radiation Therapy and Other Treatment Modalities}

One disadvantage with radiotherapy as definitive management of prostate cancer is the lack of pathologic analysis. Pathology is a strong tool used to help predict which patients may need additional interventions and when salvage treatments should be administered. Current post-radiation therapy outcome measures lack pathologic analysis and must rely only on PSA response as a biochemical predictor of prostate cancer death. Defining PSA response after radiation treatments has been difficult because the expected level, or nadir, as well as the time to nadir can vary widely between patients. Because the prostate remains intact, normal prostate cells can still produce a "background" level of PSA, which complicates the interpretation compared with the more clearly defined "undetectable" level, expected after surgery.

\section{Defining PSA Nadir following radiation}

A PSA nadir is defined as the low point to which the PSA falls after therapy. This level is not an absolute and can be highly variable between patients. In fact, the PSA nadir can fluctuate. As long as the nadir remains consistent, then radiation therapy is measured as a success. So in other words, the measure of outcome with radiation therapy is a stable drop in PSA below the pretreatment level.

There are classically two accepted definitions of biochemical relapse utilizing the PSA nadir following radiation therapy. The older ASTRO definition required three consecutive rises in PSA above the nadir to define recurrence. The date of the recurrence was considered halfway between the nadir and the date of the first rise [86]. The application of this definition was difficult in the clinical setting for several reasons to include variability in routine follow up intervals, differences in laboratory assay sensitivities and because PSA often fluctuates between values. Significant rises may be interspersed with occasional decreases of little clinical significance. Some PSA failures take a "saw tooth" upward trend in which larger rises are followed by small declines.

A second consensus panel came up with an easier, Phoenix definition which simply deems a PSA > $2 \mathrm{ng} / \mathrm{mL}$ above the nadir as a biochemical recurrence. The date of recurrence is the time the rise in PSA is noted [87].

Many papers have found significance in predicting biochemical freedom of disease based on specific nadir thresholds [88-91]. If the PSA nadir is below the threshold which predicts for a positive biochemical control rate, one interprets that the treatment was successful and the patient is biochemically disease free. If the PSA nadir is above the predictive threshold, but is remaining consistent and not rising, then the treatment is still deemed successful and the patient remains NED; however, the likelihood of remaining NED is reduced [88-91]. These thresholds have ranged from 0.2 to as high as $4.0 \mathrm{ng} / \mathrm{mL}$ [88]. A multi-institutional study was performed in which data on 4833 patients treated with definitive radiation therapy without androgen suppression were analyzed. PSA nadir groups included 0.0-0.49, 0.5-0.99, 1.0- 1.99, and $>/=2.0$. The 8 year PSA disease free survival rate was $75 \%, 52 \%, 41 \%$, and $18 \%$ respectively [88].

In addition to threshold of PSA nadir, time to nadir has been previously reported to affect outcomes [92-95]. In the same multi-institutional study just mentioned, their results showed that longer times to nadir actually predicted improved PSA disease free survival. Cut off points of 0-5.9, $6.0-11.9,12.0-23.9$ and $>24$ months showed the $8 \mathrm{yr}$ PSA disease free survival of $27 \%, 32 \%, 42 \%$, and $74 \%$ respectively [88]. In fact, some patients in this study actually did not reach PSA nadir for $>6$ yrs.

\section{Brachytherapy}

PSA nadir following interstitial brachytherapy for prostate cancer may also exhibit similar kinetics. A retrospective study from Mount Sinai medical center of over 900 patients receiving brachytherapy found that PSA nadirs of $<0.5 \mathrm{ng} / \mathrm{mL}$ were associated with an improved 5 year freedom from biochemical failure of $23.8 \%$ (95.2\% vs. $71.5 \%$ ) compared with PSA nadirs above $0.5 \mathrm{ng} / \mathrm{mL}$. This study also showed significance in time to nadir; however, their analysis predicted improved freedom from biochemical relapse when PSA nadir was achieved in less than 5 yrs of $15.9 \%$ compared with PSA nadir occurring greater than 5 yrs after treatment (96.7\% vs. 80.8\%) [96]. Zelefsky et al. in a multi-institutional study of long term outcomes for prostate cancer treated with permanent seed implant showed similar results at 8 years with a declining PSA relapse free survival for PSA nadir above $0.5 \mathrm{ng} / \mathrm{mL}$ [97].

PSA nadir is an important clinical outcome measure after radiation therapy because the nadir forms the basis from which relapse is defined. PSA bounce, a transient rise in PSA that does not represent recurrence and is temporary, will be discussed in the next section on challenges of monitoring treatment response.

\section{Cryoablation}

No clear consensus exists for the definition of biochemical failure after primary cryoablation of 
prostate cancer. Undetectable PSA, while often achievable is not typically the goal as some benign tissue will usually remain viable after the procedure. One yardstick for short-term success is PSA nadir, but there are at least two acceptable levels considered to be successful. Ellis demonstrated that $84 \%$ achieved nadir $\leq 0.4 \mathrm{ng} / \mathrm{ml}$ while Hubosky et al demonstrated successful treatment in $93 \%$ using $\leq 0.4$ as the measure of success and using a stricter criteria of nadir $\leq 0.1$, $82 \%$ reached nadir $[98,99]$. Other measures of success include using ASTRO criteria of 3 successive rises above nadir. Bahn et al reported 7 year outcomes using a combination of second and third generation cryoablation equipment and found success rates of about 90\% [100]. More recently, Pitman et al found that using the Phoenix criteria of nadir $+2 \mathrm{ng} / \mathrm{ml}$ was the most predictive of biopsy-proven local failure [101]. While randomized prospective trials including cryoablation with other forms of primary treatment for prostate cancer are lacking, these retrospective reports indicate that cryoablation can be successful and success rates, while not directly comparable seem to be reasonable.

\section{Challenges in Monitoring Treatment Response after Radiation Therapy}

One of the most difficult challenges of monitoring treatment response after radiation therapy is the phenomenon known as PSA bounce. This can occur after any form of radiation therapy and is characterized by a transient rise of PSA in the absence of recurrent disease. It can be observed in up to $>80 \%$ of patients treated with radiation if no limits are placed on its definition [102]. When PSA bounce is restricted to a specific rise then the number of those that experience it is reduced dramatically. In the same multi-institutional analysis previously mentioned in which external radiation was the treatment modality, PSA bounce was defined as a minimum rise of 0.4 $\mathrm{ng} / \mathrm{mL}$ over a period of 6 months. The bounce phenomenon occurred in 978 patients (20\%). Of those patients that experienced a bounce, approximately one quarter of them experienced two bounces. The effect of experiencing a bounce predicted for an increased risk of biochemical failure by approximately $14 \%$ (58\% vs. $72 \%)$ at 10 years [88]. This is not the case in other reported analysis in which the bounce phenomenon was associated with improved biochemical outcomes [103].

The difficulty with the bounce phenomenon is trying to distinguish it from biochemical progression. With time, a bounce will declare itself and return to nadir however the elevation can last for a year or longer. Additionally, some bounce magnitudes may actually exceed the threshold for defining biochemical failure $[103,104]$. It is important to reassure the patient and practitioner not to assume a PSA rise is evidence of recurrent or persistent disease. With newer imaging modalities on the horizon, this distinction may become less problematic; however, currently, imaging advances have not developed to a point that can reliably measure treatment response.

An additional concern regarding the monitoring of patients following radiation therapy is the contribution of benign and malignant elements to serum PSA. In a study of prostate tissue following radiation therapy, no cases of recurrent tumor with marker negative tissue were identified. However, benign prostate cells appear to sustain enough damage to lose the capacity to produce certain proteins. This may explain the lower than anticipated serum PSA levels in some patients who progress after radiation [105].

The lack of imaging to accurately measure treatment response is yet another challenge with prostate cancer. Although ultrasound, CT scans and MRI's have all been used in one form or another to attempt to detect extent of disease, they all lack sensitivity and specificity to be reliable. Advancements in imaging of prostate cancer are an area of great interest.

\section{Current Approaches in Monitoring Treatment Response in Metastatic Disease}

According to the NCCN guidelines, the frequency of clinical monitoring is determined by the response to initial androgen deprivation therapy, radiation therapy or systemic therapy [2]. Follow-up evaluation of these patients should include a history and physical examination, DRE and PSA determination every 3 to six months.

PSA remains a useful tool in monitoring treatment response in metastatic disease. PSA progression in metastatic disease has been recognized to herald clinical progression [106]. Thus, therapeutic decisions are influenced by an increasing PSA. PSA remains an integral part of monitoring treatment response in metastatic disease because it is used to identify castration-resistant disease. One of the first clinical presentations of castration-resistance occurs in a patient with a rising PSA despite medical or surgical castration. This is typically defined as a patient with a rising PSA and no radiologic evidence of metastatic prostate cancer. The Prostate Cancer Clinical Trials Working Group 2 defines PSA failure as a rising PSA that is greater than $2 \mathrm{ng} / \mathrm{mL}$ higher than the nadir; the rise has to be at least $25 \%$ over nadir and the rise has to be confirmed by a second PSA at least three weeks later [107]. In addition, the patient is required to have castrate levels of testosterone $(<50 \mathrm{ng} / \mathrm{mL})$ and no radiographic evidence of metastatic disease. 
Currently the role of imaging in monitoring treatment response in prostate cancer is not well established and there are currently no widely accepted guidelines that exist to recommend how often to image or what image modality to use in the setting of metastatic prostate cancer. The primary use of imaging techniques in advanced/metastatic prostate cancer currently is in the evaluation of new symptoms. Bone scans are readily used, but in the setting of a positive scan other modalities such as plain films, CT scans or MRI are usually used to correlate the findings.

\section{Current Challenges in Monitoring Treatment Response in Metastatic Disease}

PSA was first introduced as a way to monitor response to treatment in prostate cancer. The main advantage of PSA testing is the high sensitivity of the marker. However, given the normal expression of PSA in benign conditions such as benign prostatic hyperplasia and prostatitis it has a lower specificity. In high-risk and metastatic disease often times a very aggressive prostate cancer may have a discordantly low PSA. A prostate cancer cell population has been identified that harbors self-renewing long-term tumor-propagating cells that are refractory to stresses including androgen deprivation which may be a source of castration-resistant prostate cancer cells [108]. Thus, there may be circumstances in which PSA is potentially an inadequate way to monitor treatment response in very advanced disease.

Bone is the most common site of metastasis in prostate cancer accounting for up to $90 \%$ cases [109]. Imaging treatment response in metastatic prostate cancer has been difficult. While bone scans remain the mainstay of diagnosis of initial spread to bone, most guidelines currently only recommend them in symptomatic men or in asymptomatic men with a PSA of $>$ $20 \mathrm{ng} / \mathrm{ml}[2,6]$. Because bone scans image the osteoblastic effect of the cancer on the skeleton and not tumor proliferation, false positives may occur from non-prostate cancer-related conditions making monitoring treatment response challenging [110]. Furthermore, bone turnover, as a result of chemotherapy or androgen deprivation therapy, may increase for a period of time [111]. The sensitivity of bone scans in this setting is questionable and can lead to misdiagnosis of new metastasis. FDG-PET, the most common form of PET, uses the tracer ${ }^{18}$ F-flouro-2-deoxy-2-D-glucose (FDG) which is an indicator of glycolytic activity in cancer cells. Cancer cells have increased metabolism and utilize the less-efficient glycolytic pathway which leads to increased glucose-analogue uptake [112]. However, the use of FDG-PET imaging in prostate cancer has two major drawbacks. First, prostate cancer has a low metabolic glucose activity and second, urinary excretion of FDG results in high bladder activity that can mask tumor [113]. The sensitivity of FDG-PET for detecting prostate cancer metastatic to bone varies between 18 and 75\% [114].

\section{Future Directions in Monitoring Treat- ment Response}

\section{Active Surveillance}

Optimizing the selection criteria for men best-suited for active surveillance is currently being debated. Biomarkers have been shown to possess many important applications including use as a diagnostic to help diagnose, as a tool for determining extent of disease, for monitoring disease response to an intervention and as an indicator for prognosis [115]. The most useful biomarkers to be applied in Active surveillance will be those with the ability to determine extent of disease to decide if active surveillance is appropriate and those that determine disease progression to help determine if definitive treatment should be pursued. While potential biomarkers are not difficult to identify by "mass" assays in the lab, it will take time for any of these to be verified in clinical practice. While the prospect of these potential markers is exciting, caution and patience should always be employed when evaluating these potential additions to our prostate cancer armamentarium.

\section{Prostate Cancer Antigen 3 (PCA3)}

Prostate cancer antigen 3 (PCA3) is currently being investigated as a potential criterion for active surveillance. PCA 3 is a gene that expresses a non-coding RNA. It is only exclusively expressed in prostate tissue and is highly overexpressed in prostate cancer $[116,117]$. PCA3 detection has been shown to be associated with serum PSA levels, which showed 28-fold higher chances for cancer occurrence, with $92 \%$ specificity and $94 \%$ positive predictive value [118]. Ploussard et al prospectively studied 106 consecutive low-risk prostate cancer patients who underwent PCA3 urine test prior to prostatectomy [119]. The PCA3 score was strongly correlated with the tumour volume in a linear regression analysis $(\mathrm{p}<0.001$, $\mathrm{r}=0.409$ ). The risk of having a cancer $\geq 0.5 \mathrm{~cm}$ and a significant prostate cancer was increased three-fold in men with a PCA3 score of $\geq 25$ compared with men with a PCA3 score of $<25$ with most active surveillance biopsy criteria used. There was a trend towards higher PCA3 scores in patients with unfavourable and non-organ-confined disease and Gleason $>6$ cancers. In a multivariate analysis taking into account each 
active surveillance criterion, a high PCA3 score $(\geq 25)$ was an important predictive factor for tumour volume $\geq 0.5 \mathrm{~cm}$ and significant prostate cancer. PCA3 may, in the future have utility as a tumor marker to further improve our selection criteria for choosing men who are appropriate candidates for active surveillance.

\section{Glutathione S-transferase PI (GSTPI)}

When monitoring patients under active surveillance, if the PSA increases it is important to know if that increase is a result of progressing prostate cancer or an increase in benign prostatic hypertrophy (BPH). Glutathione S-transferase P1 is a member of an enzyme family that conjugates reactive substrates with reduced glutathione and are involved in detoxification and protecting cells from oxidative stress [120]. GSTP1 is very sensitive in detecting prostate cancer and prostatic intraepithelial neoplasia and its increased hypermethylation in neoplastic events can consistently distinguish between cancer and $\mathrm{BPH}$ [121]. If it increases during a surveillance program, this may be another indication to consider treatment.

\section{Prostate-specific Membrane Antigen (PSMA)}

Prostate-specific membrane antigen (PSMA) is a cell surface membrane. It is a type II integral membrane protein that remains cytosolic in normal prostate cells, but has been shown to translocate to the plasma membrane in prostate cancer cells [122]. PSMA levels are higher in prostate cancer and metastatic disease and appear to be up-regulated in patients with prostate cancer subsequent to androgen deprivation therapy [123]. The downside to PSMA as a biomarker is that it seems to increase with increasing age potentially decreasing its sensitivity [124].

\section{Sarcosine}

Sarcosine (N-methyl glycine) is a ubiquitous $\mathrm{N}$-methyl derivative of glycine. It was found in 2009 that levels were increased in the urine and blood in invasive prostate cancer cell lines and it was postulated that it may stimulate the growth of benign prostate cells [125]. It was also shown that by inhibiting the enzymatic step which catalyzes the production of sarcosine from glycine, prostate cancer cells were less likely to be invasive. Furthermore, up-regulating this enzymatic step increased the aggressiveness of the disease [126].

\section{Imaging}

It is estimated that between 25 to $84 \%$ of patients treated for prostate cancer (PCa) will not succumb to their disease should they be left untreated. In other words, they have a clinically insignificant form of the disease [127-129]. Therefore, in order to help discern between patients with insignificant disease and pa- tients in need of treatment, a sensitive and specific imaging modality is needed.

For many years, magnetic resonance imaging (MRI), supplemented by MR spectroscopic imaging (MRSI) has been used in the diagnosis and staging of prostate cancer. The internal anatomy of the prostate demonstrates excellent differential soft tissue contrast on T2-weighted images. The signal intensity of the normal peripheral zone is very high on $\mathrm{T} 2$ because there is higher water content there. If the prostate cancer is in the peripheral zone, a low signal intensity focus is always present on T2-weighted image, resulting in a high sensitivity of detection. Unfortunately, the specificity of the low T2 signal foci in the peripheral zone is not great (54-84\%) since these low signal foci may be seen in patients with focal infection, inflammation, bleed or post treatment changes $[130,131]$. MRSI is a MR technique that is capable of detection and quantification of normal and cancer related chemical compounds in the prostate gland. Normal prostate contains a large amount of citrate and a small amount of choline. In contrast, prostatic adenocarcinoma contains a low level of citrate and a large quantity of choline. Therefore, MRSI provides information which helps to differentiate normal prostatic tissue or other diseases from adenocarcinoma, resulting in an improved specificity. However, combination of T2WI and MRSI in the diagnosis of prostate cancer only achieves sensitivity $68 \%$ to $73 \%$ and specificity $70 \%$ to $80 \%[131,132]$. For low volume and low grade prostate cancer, the accuracy is even lower.

Recent advances of MR techniques have made prostate MR imaging much different than it was 5 or 10 years ago. This improvement is mainly attributed to the addition of diffusion weighted imaging and dynamic contrast enhanced imaging (perfusion) to the prostate MR protocol. Diffusion-weighted MR imaging is a powerful clinical tool, as it allows assessment of tissue cellularity. In prostate cancer, the cellularity in the lesion is very high, which limits the movement of water molecules. Therefore, in a patient with prostate cancer, the lesion usually appears dark on apparent diffusion coefficient (ADC) maps and normal tissue appears bright [133,134]. Furthermore, ADC values correlate with Gleason scores. Dynamic contrast-enhanced MRI (DCE-MRI) is a valuable tool in providing a map of blood flow of the prostate gland, which is increased with more vascular permeability in prostate cancer. DCE-MRI consists of a series of axial T1WI gradient echo sequences covering the entire prostate during and after IV bolus injection of gadolinium-based contrast medium. In recent studies, DCE-MRI of the prostate gland has been shown to provide excellent information of enhancement that is 
useful for both the detection and the staging of prostate cancer $[135,136]$. Because all the MR imaging techniques we have discussed have their strengths and shortcomings, they are combined in a multiparametric MR imaging (mp-MRI) prostate examination to increase accuracy. Therefore, a multiparametric MR imaging prostate examination is a MR technique with T1- and T2-weighted imaging combined with one or more functional MR imaging (DWI/ADC, DCE and MRSI). Recent study has demonstrated that combination of the three MR imaging will achieve a sensitivity of $96 \%$ and specificity of $96 \%$ in the detection of prostate cancer [137].

Predicting insignificant prostate cancer is important in the identification of patients who are ideal for active surveillance (AS). Since the diagnostic accuracy of prostate cancer at mp-MRI is very high $(>90 \%)$, this imaging technique has been increasingly used in patients on AS. First, mp-MRI can help in selecting AS candidates. Several reports have been published using DWI/ADC to estimate the aggressiveness of a tumor $[138,139]$. If mp-MRI demonstrates no clinically significant or aggressive tumor, the patient will be an ideal candidate for AS. In addition, clinical
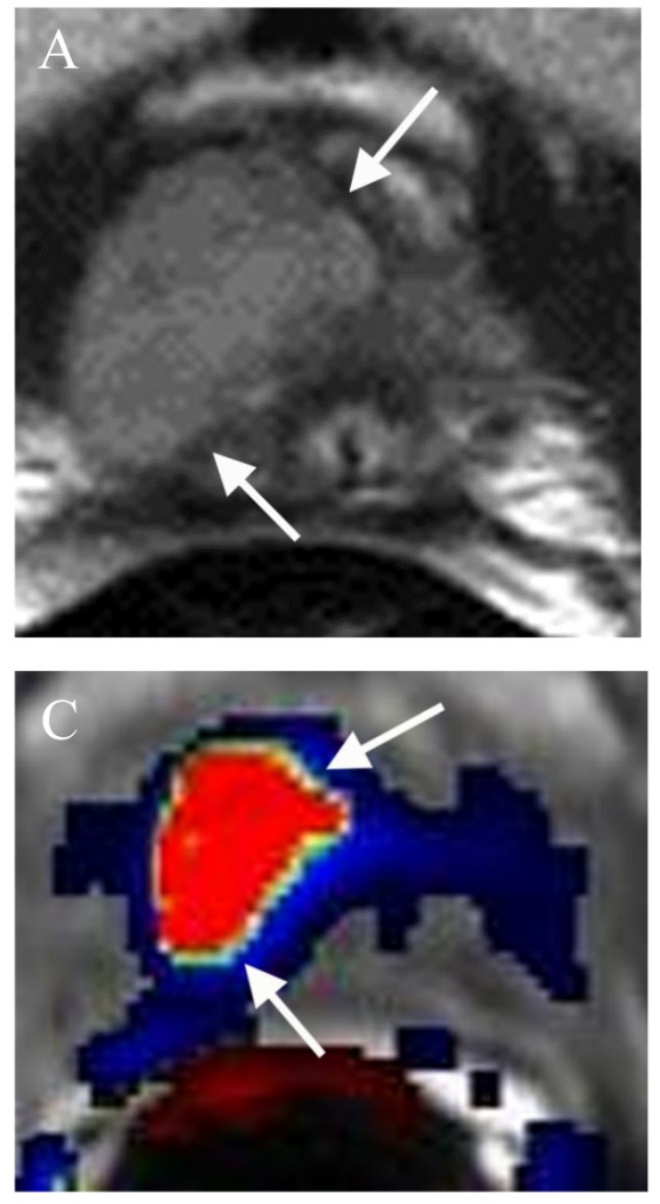

Figure I. Large lesion at the right apex missed by TRUS biopsy in a patient for pre-active surveillance screening. (a) Axial T2WI shows a large well defined mass at the right apex (arrows). (b) ADC map demonstrates the mass with low ADC value (arrows). (c) DCE shows the mass with rapid contrast wash-in and -out (red color) of the mass (arrows). (d) MRSI shows elevated choline peaks of the mass (arrows). Surgery confirmed PCa Gleason score 8. low-risk patients may still harbor significant disease, not detected by TRUS biopsy (Figure 1). Up to $35 \%$ of men on active surveillance (AS) for clinically localized prostate cancer will experience biopsy reclassification during follow-up [140]. However, a non-suspicious MRI is highly correlated with a lack of path-index lesions in an AS population [140]. Second, mp-MRI can provide serial information of the tumor under active surveillance. If a low grade prostate tumor can be identified at mp-MRI, a follow- up (likely 12 months) mp-MRI will provide information of the size and appearance of the lesion (Figure 2). If the lesion is stable, the patient will likely continue to stay on AS. In contrast, trans- rectal ultrasound (TRUS) guided prostate biopsy for patients on AS has been suboptimal because the yearly biopsy results for these patients may be very different, creating a lot of confusion and frustration among physicians and patients [141]. If the lesion on follow-up mp-MRI shows an increase in size and / or aggressiveness, MRI directed TRUS or MRI guided prostate biopsy can be performed. The result of the biopsy will guide the future direction of the patient's treatment.
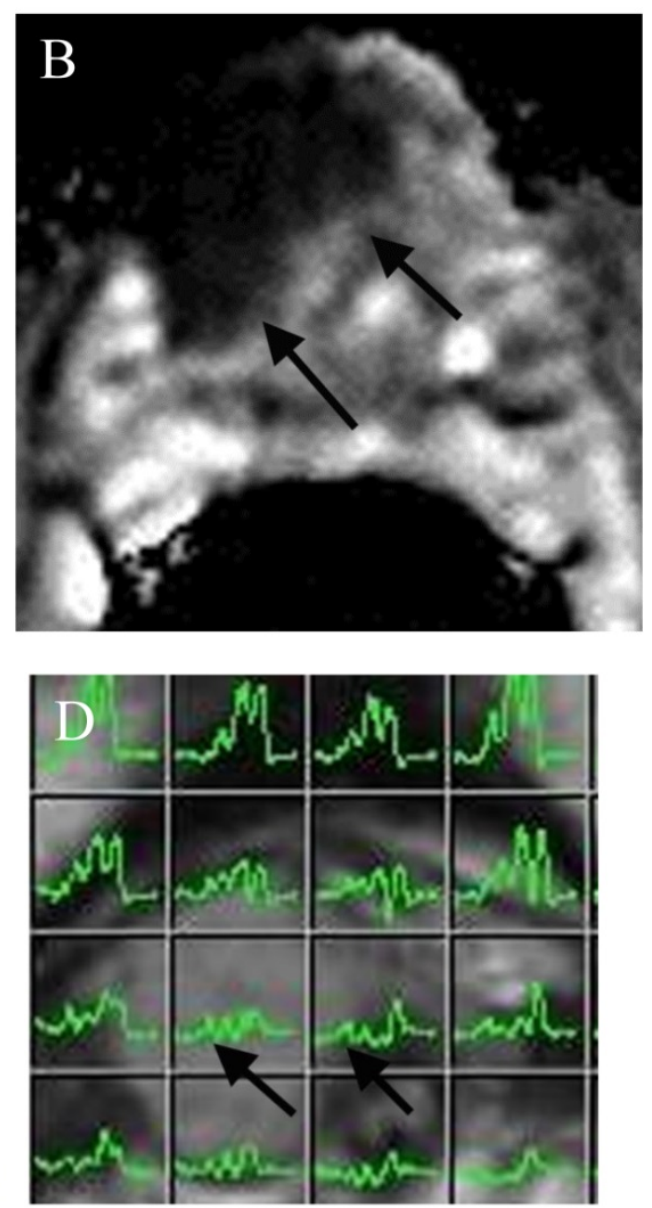

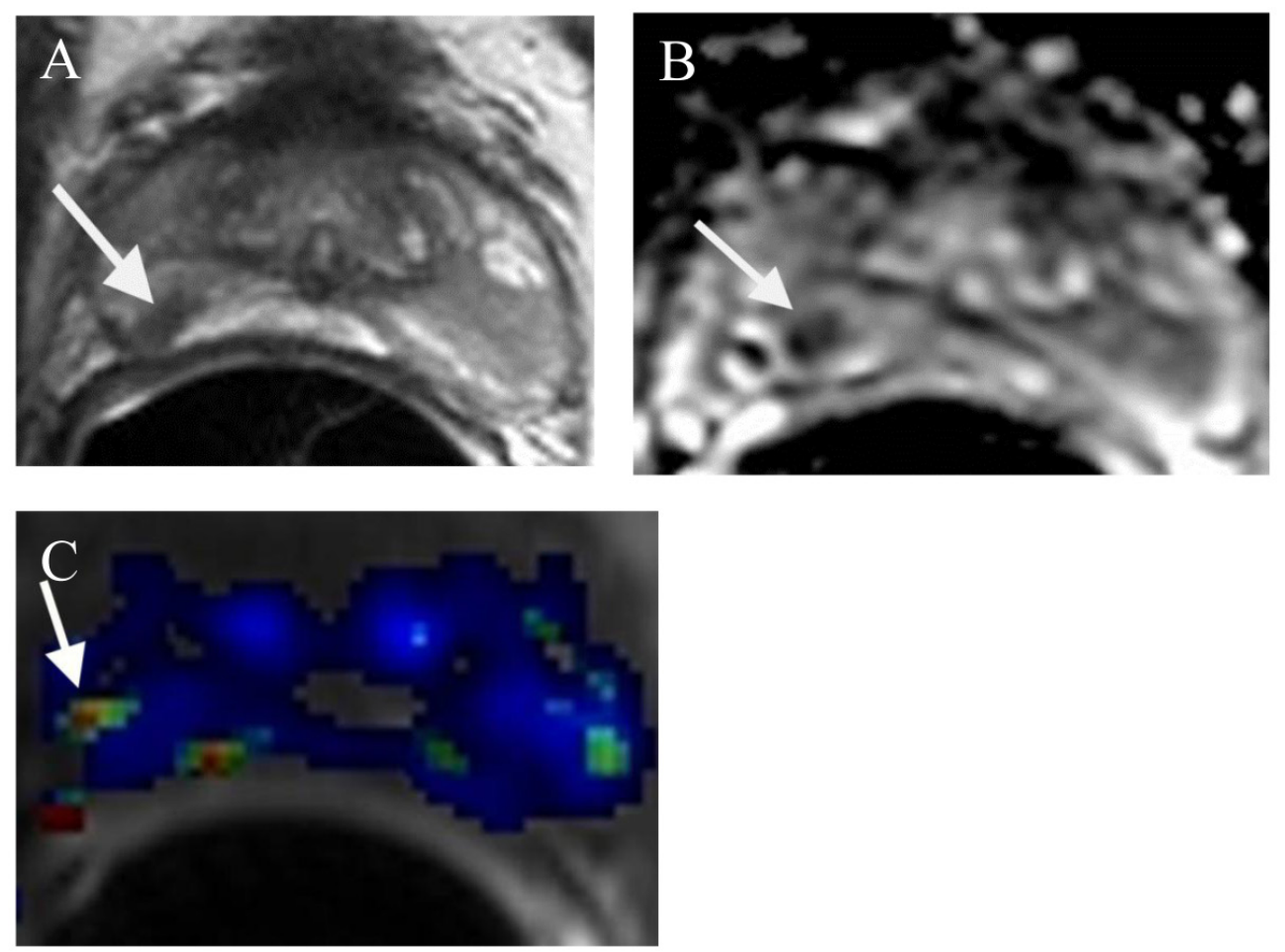

Figure 2. A small prostate cancer Gleason score 6 at the right mid PZ in a patient for AS. (a) (a) Axial T2WI shows a small low T2 signal intensity lesion at the right mid (arrow). (b) ADC map demonstrates a small low ADC signal intensity lesion at the right mid (arrow). (c) DCE demonstrates a small lesion at the right mid with contrast wash in and out (arrow).

\section{Surgery and Radiation Therapy}

\section{Supersensitive PSA}

Although the ultrasensitive PSA assays (Third generation) that are currently common, measure PSA to $0.01 \mathrm{ng} / \mathrm{mL}$, the presence of prostate cancer still can be missed. Newer tests are enabling earlier cancer detection with $4^{\text {th }}$ and $5^{\text {th }}$ generation assays measuring down to $0.00005 \mathrm{ng} / \mathrm{mL}$ [142]. The question will be what to do with this information once it is available. Initiating salvage therapies at these levels has not been reported but the possibility provokes interest and further study.

\section{Genomic Predictors on the Horizon}

Intermediate risk prostate cancer is problematic because of the varied response to treatment. Unlike low risk and high risk disease, intermediate risk prostate cancer can often be under or over treated. Novel biologic and genetic prognosticators are being investigated to help individualize therapy. The University of Toronto and the Ontario Cancer Institute/Princess Margaret Hospital found significant genetic alterations in prostate cancer biopsy specimens of c-MYC, PCSA, and NKX3.1 of which the NKX3.1 alone and in concert with c-MYC was significantly associated with biochemical relapse free rate [143].

\section{New Potential Markers}

Most solid tumors possess cellular abnormalities that involve either the nuclear and/or extracellular matrix. Periostin a component of the extracellular matrix, is a protein produced by fibroblasts. Periostin (POSTN) can act as a tumor promoter or as a tumor suppressor gene and is currently being studied in the context of prostate cancer. High stromal expression was associated with increased extracapsular extension on prostatectomy specimens and a shorter survival. However, very low POSTN scores were associated with a shorter PSA-free survival as well, which supports its role as both a promoter and suppressor gene [144]. Further understanding of this interesting marker may play a role in customizing prostate cancer treatment.

Imaging

Local recurrence of prostate cancer after radical prostatectomy (RP) is very frequent, affecting 20-50\% of patients after RP during a 10-year follow-up $[145,146]$. When PSA relapse has been diagnosed, it is essential to determine whether the recurrence has developed at distant or local sites for the relevant treatment planning; hormone therapy for systemic disease and external beam radiation therapy (EBRT) for local recurrence. Recently mp-MRI has emerged as an important diagnostic tool in detecting local recur- 
rence of prostate cancer after RP. Dynamic contrast-enhanced magnetic resonance imaging (DCE-MRI) has proved to be the most reliable technique in detecting local recurrence, even though T2WI is necessary for every MR study (Figure 3) and DWI can be proposed as a reliable alternative $[147,148]$. The use of mp-MRI is likely to increase in this clinical setting every year, as there are no good alternative modalities currently available.

Radiation therapy is one of the major treatment options for achieving curative treatment of local disease. Thirty percent of cases treated with radiation therapy will suffer biochemical failure within 5 years of completing therapy [149]. However, biochemical recurrence does not differentiate local recurrence from systemic disease. Since local recurrence may be amenable to salvage prostatectomy, salvage brachytherapy or newer minimally invasive approaches such as cryotherapy or high-intensity focused ultrasound, this distinction between local recurrence and systemic disease is crucial. As we all know that the nonspecificity of PSA fluctuations for early recurrence is very common, a reliable imaging technique is of particular value if it is capable to detect local recurrence. Many recent studies show a great role for $\mathrm{mp}-\mathrm{MRI}$ in detecting local recurrence following external beam radiation therapy or brachytherapy [150,151], Because radiation-induced anatomic changes such as diffuse T2 signal hypointensity in the gland and indistinctness of the normal zonal anatomy limit the utility of conventional

morphologic evaluations with T2-weighted MRI, functional prostate MRI (DWI, DCE and MRSI) has been proven repeatedly to be an accurate imaging technique in detecting local recurrence [150-152]. Furthermore, mp-MRI can also be used in monitoring the response following focal salvage brachytherapy (Figure 4).

\section{Metastatic Disease}

Imaging

The future application of PET in the monitoring of treatment response in metastatic prostate cancer will utilize new tracers more specific to prostate such as antibodies to such prostate specific molecules as prostate-specific membrane antigen. Gene expression imaging and imaging of cell trafficking during adoptive immunotherapy will be developed. Currently, new tracers are being investigated. ${ }^{11} \mathrm{C}$-methionine differentiates tumor from normal tissue due to elevated protein synthesis [153]. New choline and acetate tracers are also being investigated including 11C-acetate, 11C-choline and 18F-choline which have shown promise in imaging prostate cancer metastases [154].

\section{Biomarkers}

Biomarkers have been shown to possess many important applications including use as a diagnostic to help diagnose, as a tool for determining extent of disease, for monitoring disease response to an intervention and as an indicator for prognosis [115]. The most useful biomarkers to be applied in metastatic disease will be those with the ability to determine or predict castration-resistance, aggressiveness of disease and response to treatment.
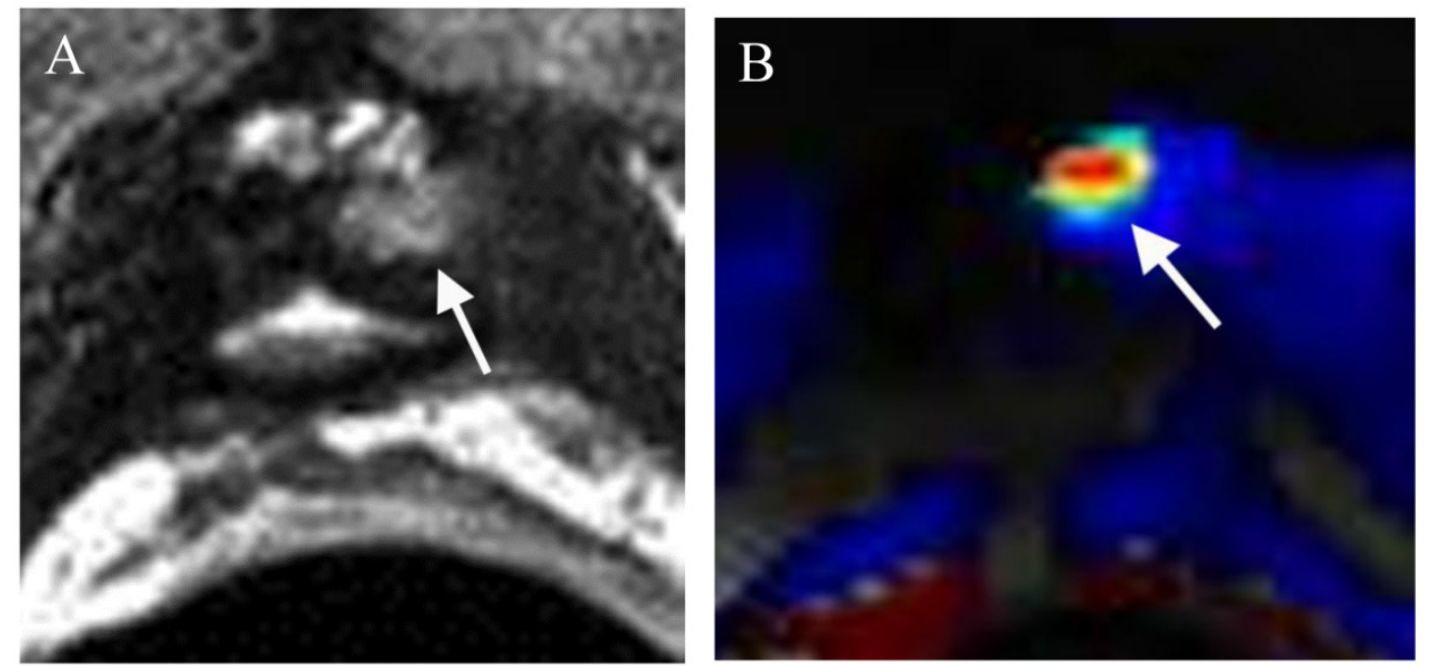

Figure 3. Small recurrence in the left prostate surgical bed in a patient status post radical prostatectomy with a recent elevation of PSA to $0.2 \mathrm{Ing} / \mathrm{ml}$. (a) Axial T2WI shows a small mass with slightly high T2 signal (arrow) relative in the left prostate surgical bed. (b) DCE shows the lesion with rapid contrast wash-in and -out (arrow) consistent with a focal recurrence. 

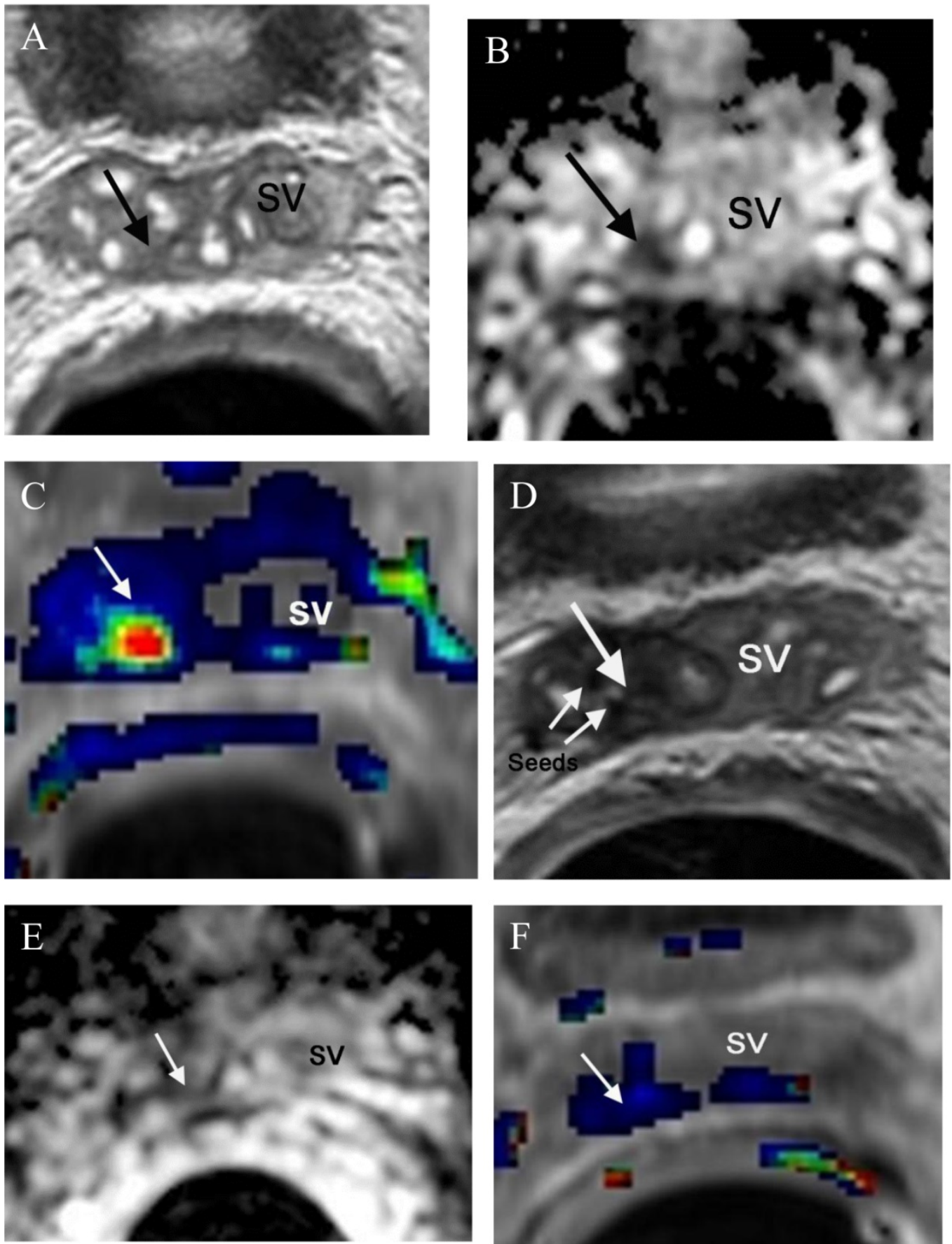

Figure 4. Small recurrence in the right seminal vesicle (confirmed by MRI guided biopsy) in a patient status post internal radiation seeds treatment with an elevation of PSA to $2.7 \mathrm{ng} / \mathrm{ml}$. Focal salvage brachytherapy was performed later on. (a) Axial T2Wl shows a small low T2 signal intensity lesion (arrow) in the right seminal vesicle (SV). (b) ADC map demonstrates a small low ADC signal intensity lesion (arrow) in the right seminal vesicle (SV). (c) DCE shows the lesion with rapid contrast wash-in and -out (arrow) in the right seminal vesicle (SV). (d) Axial T2Wl shows the small low T2 signal intensity lesion (arrow) surrounded by radiation seeds (seeds) in the right seminal vesicle (SV). (e) ADC map demonstrates no diffusion restriction at the site of the lesion (arrow) in the right seminal vesicle (SV). (f) DCE shows the lesion with disappearance of abnormal contrast enhancement (arrow) in the right seminal vesicle (SV) after focal salvage brachytherapy.

\section{Plasma miRNAs}

MicroRNAs are key regulators of many biological processes and may be used as clinical marker of disease. In one recent study, 10 prospective miRNA candidates were identified as potential biomarkers to differentiate between localized prostate cancer and metastatic castration resistant prostate cancer [155]. While no single miRNA alone differentiated the two disease states, combinations had greater sensitivity and specificity indicating the potential of a future RNA assay that may help us clinically determine those at-risk for castration resistant disease. 


\section{APC methylation}

A study evaluating 218 human prostate tissue samples (106 benign prostatic hyperplasia and 112 prostate cancer) were assessed and the methylation of adenomatous polyposis coli (APC) was analyzed by quantitative pyrosequencing [156]. The sensitivity and specificity of APC methylation status differentiating between prostate cancer and $\mathrm{BPH}$ reached $89.3 \%$ and $98.1 \%$, respectively. The APC methylation level correlated positively with Gleason grade.

\section{Bone Turnover Markers (BAP and uNTX)}

The bone-forming metastases of prostate cancer are the result of complex interactions within the microenvironment. Autocrine-paracrine signaling pathways between prostate cancer epithelium, osteoblasts and osteoclasts stimulate aberrant bone remodeling and it is postulated that these interactions can be quantitatively measured using bone-specific alkaline phosphatase (BAP) and urine $\mathrm{N}$-telopeptide (uNTX) [157]. It was shown that baseline levels of BAP were prognostic in both androgen-dependent and castrate-resistant disease. A reduction in BAP, $\mathrm{uNTX}$ or BAP/uNTX on therapy was predictive of overall survival in both patient groups.

\section{Conclusions}

The current state of monitoring treatment response in prostate cancer has not fundamentally changed since the incorporation of PSA as a biomarker in clinical use in the 1980s. While there are challenges with our current approaches, especially with regard to imaging this disease, we seem to be standing on a precipice with regard to both imaging and biomarkers that will soon take us to a new level in our ability, to not only monitor treatment response, but also to enable us to choose the most appropriate treatment. Knowing when not to treat prostate cancer is just as important as knowing which treatment to choose and we are developing tools that will aid us in choosing the right path for our patients.

\section{Competing Interests}

The authors have declared that no competing interest exists.

\section{References}

1. [Internet] Howladder N, Noone AM, Krapcho M, Garshell J, Neyman N, Altekruse SF, Kosary CL, Yu M, Ruhl J, Tatalovich Z, Cho H, Mariotto A, Lewis DR, Chen HS, Feur EJ, Cronin KA. SEER Cancer Statistics Review, 1975-2010, National Cancer Institute. Bethesda, MD. http://cancer.gov/csr/1975_2010/

2. [Internet] National Comprehensive Cancer Network (NCCN). Clinical practice guidelines in oncology: prostate cancer. http://www.nccn.org.

3. Alberta Provincial Genitourinary Tumour Team. Prostate cancer. Edmonton (Alberta): Alberta Health Services, Cancer Care. 2011. (Clinical practice guideline; no. GU-004).

4. [Internet] http://www.nice.org.uk/CG058
5. Thompson I et al. Guideline for the management of clinically localized prostate cancer: 2007 update. J Urol. 2007;177(6):2106-2131.

6. Heidenreich A et al. Guidelines on Prostate Cancer. Eur Urol. 2008;53(1):68-80.

7. Wilt TJ, MacDonald R, Rutks I et al. Systematic Review: comparative effectiveness and harms of treatments for clinically localized prostate cancer. Ann Intern Med. 2008;148:435-448.

8. Bolla M, Collette L, Blank L, et al. Long-term results with immediate androgen suppression and external irradiation in patients with locally advanced prostate cancer (an EORTC study): a phase III randomized trial. Lancet. 2002;360:103-106.

9. Hanks GE, Pajak TF, Porter I, et al. Phase III trial of long-term adjuvant androgen deprivation after neoadjuvant hormonal cytoreduction and radiotherapy in locally advanced carcinoma of the prostate: the Radiation Therapy Oncology Group Protocol 92-02. J Clin Oncol. 2002;21:3972-3978.

10. Bolla M, de Reijke TM, Van Tienhoven G, et al. Duration of androgen suppression in the treatment of prostate cancer. N Engl J Med. 2009;360:2516-2527.

11. Roach M, DeSilvio M, Lawton C, et al. Phase III trial comparing whole-pelvic versus prostate-only radiotherapy and neoadjuvant versus adjuvant combined androgen suppression: Radiation Therapy Oncology Group 9413. J Clin Oncol. 2003;21:1904-1911.

12. Dearnaley DP, Mason MD, Parmar MK, et al. Adjuvant therapy with sodium clodronate in locally advanced and metastatic prostate cancer: long-term overall survival results from the MRC PR04 and PR05 randomised controlled trials. Lancet Oncol. 2009; 10:872-876.

13. Saad F, Gleason DM, Murray R, et al. A randomized, placebo-controlled trial of zoledronic acid in patients with hormone-refractory metastatic prostate carcinoma. J Natl Canc Inst. 2002;94:1458-1468.

14. Smith MR, et al. Toremifene to reduce fracture risk in men receiving androgen deprivation therapy for prostate cancer. J Urol. 2013;189:S45-S50.

15. Lipton A, et al. Superiority of denosumab to zoledronic acid for prevention of skeletal-related events: a combined analysis of 3 pivotal randomized phase III trials. Eur J Can. 2012;48:3082-3092.

16. Petrylak DP, Tangen CM, Hussain MH, et al. Docetaxel and estramustine compared with mitoxantrone and prednisone for advanced refractory prostate cancer. N Engl J Med. 2004;351:1513-1520.

17. Tannock IF, de Wit R, Berry WR, et al. Docetaxel plus prednisone or mitoxantrone plus prednisone for advanced prostate cancer. $N$ Engl J Med. 2004:351:1502-1512.

18. Sternberg CN, Petrylak DP, Sartor O, et al. Multinational, double-blind, phase III study of prednisone and either satraplatin or placebo in patients with castrate-refractory prostate cancer progressing after prior chemotherapy: the SPARC trial. J Clin Oncol. 2009; 27:5431-5438.

19. Kantoff PW, Higano CS, Shore ND, et al. Sipuleucel-T immunotherapy for castration-resistant prostate cancer. N Engl J Med. 2010;363:411-422.

20. Dicki GJ, et al. Strontium and samarium therapy for bone metastases from prostate carcinoma. Australas Radiol. 1999;43:476-479.

21. Parker C, et al. Updated analysis of the phase III, double blind, multinational study of radium-223 chloride in castration-resistant prostate cancer (CRPC) patients with bone metastases (ALSYMPCA). ASCO 2013 Abstract .

22. Pisters LL, Dinney CP, Pettaway CA, et al. A feasibility study of cryotherapy followed by radical prostatectomy for locally advanced prostate cancer. J Urol. 1999;161:509-514.

23. Cresswell J, Asterling S, Chaudhary M, et al. Third-generation cryotherapy for prostate cancer in the UK: a prospective study of the early outcomes in primary and recurrent disease. BJU Int. 2006;97:969-974.

24. Eggener S, Salomon G, Scardino PT, et al. Focal therapy for prostate cancer: possibilities and limitations. Eur Urol. 2010;58:57-641

25. Shelley M, Wilt TJ, Coles B, et al. Cryotherapy for localised prostate cancer. Cochrane Database Syst Rev. 2007;(3):CD005010

26. Lee HM, Hong JH, Choi HY. High-intensity focused ultrasound therapy for clinically localized prostate cancer. Prostate Cancer Prostatic Dis. 2006;9:439-443

27. Uchida $\mathrm{T}$, Ohkusa $\mathrm{H}$, Yamashita $\mathrm{H}$, et al. Five years experience of transrectal high-intensity focused ultrasound using the Sonablate device in the treatment of localized prostate cancer. Int J Urol. 2006;13:228-233

28. Uchida T, Baba S, Irie A, et al. Transrectal high-intensity focused ultrasound in the treatment of localized prostate cancer: a multicenter study. Hinyokika Kiyo. 2005;51:651-658

29. Wallace T, Avital I, Stojadinovic A, et al. Multi-parametric MRI-directed focal salvage permanent interstitial brachytherapy for locally recurrent adenocarcinoma of the prostate: a novel approach. J Cancer 2013;4(2):146-151.

30. Ahmed HU, Cathcart P, McCartan N, et al. Focal salvage therapy for localized prostate cancer recurrence after external beam radiotherapy: a pilot study. Cancer 2012;118(17):4148-55.

31. Gowardhan B, Greene D. Salvage cryotherapy: is there a role for focal therapy? J Endourol 2010;24(5):861-4.

32. Ahmed HU, Freeman A, Kirkham A, et al. Focal therapy for localized prostate cancer: a phase I/II trial. J Urol 2011;185(4):1246-54.

33. Dickinson L, Ahmed HU, Kirkham A, et al. A multicenter prospective development study evaluating focal therapy using high intensity focused ultrasound for localized prostate cancer: the INDEX study. Contemp Clin Trials 2013;:s1551.

34. Pound CR, Partin AW, Eisenberger MA, et al. Natural history of progression after PSA elevation following radical prostatectomy. JAMA 1999;281:1591-1597. 
35. Horwitz EM, Thames HD, Kuban DA, et al. Definitions of biochemical failure that best predict clinical failure in patients with prostate cancer treated with external beam radiation alone: a multi-institutional pooled analysis. J Urol 2005 Mar;173(3):797-802.

36. Coleman RE. Clinical features of metastatic bone disease and risk of skeletal morbidity. Clin Cancer Res 2006;12:6243s-6249s.

37. Hayward JL, Carbone PP, Heusen JC, Kumaoka S, Segaloff A, Rubens RD. Assessment of response to therapy in advanced breast cancer. Br J Cancer 1977;35:292-298.

38. WHO. WHO handbook for reporting results of cancer treatment. Geneva, Switzerland: World Health Organization Offset publication. 1979.

39. Therasse P, Arbuck SG, Eisenhauer EA et al. New guidelines to evaluate the response to treatment in solid tumors (RECIST Guidelines). J Natl Cancer Inst 2000;92:205-216.

40. Eisenhauer EA, Therasse P, Bogaerts J, et al. New response evaluation criteria in solid tumours: revised RECIST guidelines (version 1.1). Eur J Cancer 2009;45:228-247.

41. Hamaoka T, Madewell JE, Podoloff DA, Hortobagyi GN, Ueno NT. Bone imaging in metastatic breast cancer. J Clin Oncol 2004; 22:2942-2953.

42. Hamaoka T, Costelloe CM, Madewell JE, et al. Tumour response interpretation with new tumour response criteria vs the World Healt Orgnisation criteria in patients with bone-only metastatic breast cancer. $\mathrm{Br} \mathrm{J}$ Canc 2010;102:651-657.

43. Hayashi N, Costelloe CM, Hamaoka T, Wei C, et al. A prospective study of bone tumor response assessment in metastatic breast cancer. Clin Breast Cancer 2013;13(1):24-30.

44. Wahl RL, Jacene H, Kasamon $\mathrm{Y}$, Lodge MA. From RECIST to PERCIST: Evolving considerations for PET response criteria in solid tumors. J Nucl Med 2009;50 (suppl 1):122s-150s.

45. Scher HI, Morris MJ, Kelly WK, et al. Prostate cancer clinical trial end points: "RECIST"ing a step backwards. Clin Cancer Res 2005;11(14):5223-5232.

46. Bolger JJ, Dearnaley DP, Kirk D, et al. Strontium-89 (Metastron) versus external beam radiotherapy in patients with painful bony metastases secondary to prostatic cancer: preliminary report of a multicenter trial. Semin Oncol 1993;20:32-33.

47. Saad F, Gleason DM, Murray R, et al. A randomized, placebo-controlled trial of zoledronic acid in patients with hormone-refractory metastatic prostate carcinoma. J Natl Cancer Inst 2002;94:1458-68.

48. Klotz L, Zhang L, Lam A, et al. Clinical results of long-term follow-up of a large, active surveillance cohort with localized prostate cancer. J Clin Oncol 2010; 28(1):126-31.

49. Ross AE, Loeb S, Landis P, Partin AW, Epstein JI, et al. Prostate-specific antigen kinetics during follow-up are an unreliable trigger for intervention in a prostate cancer surveillance program. J Clin Oncol 2010; 28(17):2810-16.

50. Lawrentschuk N, Haider MA, Daljeet N, et al. "Prostatic evasive anterior tumors": the role of magnetic resonance imaging. BJU Int 2010;105(9):1231-6.

51. van den Bergh RC, Roemeling S, Roobol MJ, et al. Outcomes of men with screen-detected prostate cancer eligible for active surveillance who were managed expectantly. Eur Urol 2009;55(1):1-8.

52. Kazer MW, Psutka SP, Latini DM, Bailey DE. Psychosocial aspects of active surveillance. Curr Opin Urol 2013; 23(3):273-277.

53. Wong LM, Johnston R, Sharma N, Shah NC, Warren AY, Neal DE. General application of the National Institute for Health and Clinical Excellence (NICE) guidance for active surveillance for men with prostate cancer is not appropriate in unscreened populations. BJU Int. 2012 Jul;110(1):24-7.

54. Stephenson AJ, Scardino PT, Kattan MW, et al. Predicting the outcome of salvage radiation therapy for recurrent prostate cancer after radical prostatectomy. J Clin Oncol 2007;25:2035-41.

55. Swindler P, Eastham JA, Ohori M et al. Do margins matter? The prognostic significance of positive surgical margins in radical prostatectomy specimens. J Urol 2005; 174: 903

56. Kupelian PA, Katcher J, Levin HS et al: Stage T1-2 prostate cancer: a multivariate analysis of factors affecting biochemical and clinical failures after radical prostatectomy. Int J Radiat Oncol Biol Phys 1997; 377: 1043.

57. Lee HM, Solan MJ, Lupinacci $P$ et al: Long-term outcome of patients with prostate cancer and pathologic seminal vesicle invasion (pT3b): Effect of adjuvant radiotherapy. Urology 2004; 64: 84.

58. Ohori M, Wheeler TM, Kattan MW et al: Prog- nostic significance of surgical margins in radical prostatectomy specimens. J Urol 1995; 154: 1818.

59. Lowe BA and Lieberman SF: Disease recurrence and progression in unrelated pathologic stage $\mathrm{T} 3$ prostate cancer: selecting the patient for adju- vant therapy. J Urol 1997; 158: 1452.

60. Bolla M, van Poppel H, Tombal B et al: Postop- erative radiotherapy after radical prostatectomy for high-risk prostate cancer: long-term results of a randomized controlled trial (EORTC trial 22911). Lancet 2012; 380: 2018.

61. Thompson IM, Tangen CM, Paradelo J et al: Adjuvant radiotherapy for pathological T3N0M0 prostate cancer significantly reduces risk of metastases and improves survival: long-term follow-up of a randomized clinical trial. J Urol 2009; 181: 956.

62. Wiegel T, Bottke D., Steiner U, et al. Phase III postoperative adjuvant radiotherapy after radical prostatectomy compared with radical prostatectomy alone in $\mathrm{pT} 3$ prostate cancer with postoperative undetectable prostate-specific antigen: ARO 96-02/AUO AP 09/95. J Clin Oncol, 2009;27: 292

63. Thompson IM, Valicenti RK, Albersen P, et al. Adjuvant and salvage radiotherapy after prostatectomy: AUA/ASTRO guideline. J Urol 2013;190: 443
64. Yee DS, Shariat SF, Lowrance WT, Maschino AC, Savage CJ, Cronin AM, Scardino PT, Eastham JA. Prognostic significance of lymphovascular invasion in radical prostatectomy specimens. BJU Int. 2010;108:502-7.

65. Shariat SF, Khoddami SM, Saboorian H, Koeneman KS, Sagalowsky AI, Cadeddu JA, McConnell JD, Holmes MN, Roehrborn CG. Lymphovascular invasion is a pathologic feature of biologically aggressive disease in patients treated with radical prostatectomy. J Urol 2004; 171:1122-7.

66. May M, Kaufmann O, Hammermann F, Loy V, Siegsmund M. Prognostic impact of lymphovascular invasion in radical prostatectomy specimens. BJU Int. 2006; 99:539-44.

67. Epstein JI, Srigley J, Grignon D, Humphrey P. Recommendations for the reporting of prostate carcinoma. Human Pathology. 2007;38:1305-9.

68. Cheng L, Zincke H, Blute ML, Bergstralh EJ, Scherer B, Bostwick DG. Risk of prostate carcinoma death in patients with lymph node metastasis. Cancer 2001;91:66-73

69. Messing EM, Manola J, Sarosdy M et al. Immediate hormonal therapy vs. observations in men with node positive prostate cancer following radical prostatectomy and pelvic lymphadenectomy: a randomized phase III Eastern Cooperative Oncology Group/ Intergroup Trial. N Engl J Med 1999;341:1781-88.

70. Messing EM, Manola J, Yao J, Kiernan M, Crawford D, Wilding G, di'SantAgnese PA, Trump D. Immediate versus deferred androgen deprivation treatment in patients with node-positive prostate cancer after radical prostatectomy and pelvic lymphadenectomy. Lancet Oncol 2006;7:472-9.

71. Cookson MS, Aus G, Burnett AL, et al. Variation in the definition of biochemical recurrence in patients treated for localized prostate cancer: the American Urological Association Prostate Guidelines for Localized Prostate Cancer Update Panel report and recommendaions for standard in the reporting of surgical outcomes. J Urol 2007; 177:540.

72. Partin AW, Pearson JD, Landis PK, et al. Evaluation of serum prostate-specific antigen velocity after radical prostatectomy to distinguish local recurrence from distant metastases. Urology 1994; 43:649.

73. Moul JW. Prostate specific antigen only progression of prostate cancer. J Urol. 2000;163(6):1632-42.

74. King CR. Adjuvant versus salvage radiotherapy for high-riskprostate cancer patients. Semin Radiat Oncol. 2013 23(3):215-21

75. King CR: The timing of salvage radiotherapy after radical prostatectomy: a systematic review. Int J Radiat Oncol Biol Phys 2012;84(1):104-11

76. Stephenson A.J, et al. Predicting the outcome of salvage radiation therapy for recurrent prostate cancer after radical prostatectomy. J Clin Oncol, 2007; 25: 2035-2041

77. Trock BJ, Han M, Freedland SJ, Humphreys EB, DeWeese TL, Partin AW, Walsh PC. Prostate cancer-specific survival following salvage radiotherapy vs observation in men with biochemical recurrence after radical prostatectomy. JAMA 2008; 299: 2760-69.

78. Karlin JD, Koontz BF, Freedland SJ, Moul JW, Grob BM, Wan W, Hagan MP, Anscher MS, Moghanaki D. Identifying appropriate patients for early salvage radiotherapy after prostatectomy. J Urol 2013; 190: 1-6.

79. McGuire BB, Helfand BT, Loeb S, et al. Outcomes in patients with Gleason score 8-10 prostate cancer: relation to preoperative PSA level. BJU Int 2012; 109(12):1764-9.

80. Loeb S, Epstein JI, Ross AE, Schultz L, Humphreys EB, Jarow JP. Benign prostate glands at the bladder neck margin in robotic vs. open radical prostatectomy. BJU International 2010;105:1446-9.

81. Godoy $\mathrm{G}$, Tareen BU, Lepor $\mathrm{H}$. Does benign prostatic tissue contribute to measurable PSA levels after radical prostatectomy? Oncology 2009;74:167-170.

82. Alanen KA, Kuopio T, Koskinen PJ, Nevalainen TJ. Immunohistochemical labeling for prostate specific antigen in non-prostatic tissues. Pathol Res Pract. 1996; 192:233-7.

83. Holmes GF, Eisele DW, Rosenthal D, Westra WH. PSA immunoreactivity in a Parotid Oncocytoma: A diagnostic pitfall in discriminating primary parotid neoplasms from metastatic prostate cancer. Diagnostic Cytopathology 1998; 19: 221.

84. Elgamal AA, Ectors NL, Sunardhi-Widyaputra S, Van Poppel HP, Van Damme BJ, Baert LV. Detection of prostate specific antigen in pancrease and salivary glands: a potential impact on prstate cancer overestimation. J Urology 1996;156:464-8.

85. Mohammadizadeh F, Ranaee M, Zavareh MH, Faghihi M, Saremi M, Eftekhari $\mathrm{M}$. The expression of prostate-specific antigen in invasive breast carcinoma and its relationship with routin clinicopathologic parameter. Adv Biomed Res 2012;1:55.

86. Consensus statement guidelines for PSA following radiation therapy. American Society for Therapeutic Radiology and Oncology Consensus Panel. Int J Radiat Oncol Biol Phys 1997; 37:1035.

87. Roach M $3^{\text {rd }}$, Hanks G, Thames H Jr, et al. Defining biochemical failure following radiotherapy with or without hormonal therapy in men with clinically localized prostate cancer: recommendations of the RTOG-ASTRO Phoenix Consensus Conference. Int J Radiat Oncol Biol Phys 2006; 65:965.

88. Ray ME, Thames HD, Levy LB Horwitz EM, Kuban DA, et al: PSA nadir predicts biochemical and distant failures after external beam radiotherapy for prostate cancer: a multi-institutional analysis. Int J Radiation Oncology Biol Phys 2006;64: 1141.

89. Crook JM, Bahadur YA, Bociek RG et al. Radiotherapy for localized prostate carcinomaThe correlation of pretreatment prostate specific antigen and nadir 
prostate specific antigen with outcome as assessed by systematic biopsy and serum prostate specific antigen. Cancer, 1997; 79: 328-336.

90. DeWitt K.D, Sandler H.M, Weinberg V, et al. What does postradiotherapy PSA nadir tell us about freedom from PSA failure and progression-free survival in patients with low and intermediate-risk localized prostate cancer? Urology, 2003; 62: 492-496.

91. Hanlon A.L., Diratzouian H., Hanks G.E. Posttreatment prostate-specific antigen nadir highly predictive of distant failure and death from prostate cancer. Int J Radiat Oncol Biol Phys, 2002;53: 297-303.

92. Aref I., Eapen L., Agboola O, et al. The relationship between biochemical failure and time to nadir in patients treated with external beam therapy for T1-T3 prostate carcinoma. Radiother Oncol, 1998;48: 203-207.

93. Cavanaugh S.X., Kupelian P.A, Fuller C.D, et al. Early prostate-specific antigen (PSA) kinetics following prostate carcinoma radiotherapy: Prognostic value of a time-and-PSA threshold model. Cancer, 2004;101: 96-105.

94. Kestin LL, Vicini FA, Ziaja EL et al. Defining biochemical cure for prostate carcinoma patients treated with external beam radiation therapy. Cancer, 1999; 86: 1557-1566

95. Critz FA. Time to achieve a prostate specific antigen nadir of $0.2 \mathrm{ng} / \mathrm{ml}$ after simultaneous irradiation for prostate cancer. J Urol, 2002;168: 2434-2438.

96. Ko EC, Stone NN, Stock RG. PSA nadir of $<0.5 \mathrm{ng} / \mathrm{mL}$ following brachytherapy for early-stage prostate adenocarcinoma is associated with freedom from prostate-specific antigen failure. Int J Radiat Oncol Biol Phys. 2012;83(2):600-7.

97. Zelefsky MJ, Kuban DA, Levy LB, Horwitz EM et al. Multi-institutional analysis of long-term outcome for stages T1-T2 prostate cancer treated with permanent seed implantation. Int J Radiat Onc Biol Phys 2007;67(2):327-33.

98. Ellis DS. Cryosurgery as primary treatment for localized prostate cancer: a community hospital experience. Urol 2002;60(suppl 2a): 34-9.

99. Hubosky SG, Fabrizio MD, Schellhammer PF, Barone BB, Tepera CM, Given RW. Single center experience with third generation cryosurgery for management of organ confined prostate cancer: critical evaluation of short term outcomes, complications, and patient quality of life. J Endourol 2007;21:1521-31.

100. Bahn DK, Lee F, Baldalament R, Kumar A, Greski J, Chernick M. Targeted cryoablation of the prostate: seven year outcomes in the primary treatment of prostate cancer. Urol 2002; 60(suppl 2a):3-11.

101. Pitman M, Shapiro EY, Hruby GW, Truesdale MD, Cheetham PJ, Saad S, Katz AE. Comparison of biochemical failure definitions for predicting local cancer recurrence following cryoablation of the prostate. The Prostate 2012;72:1802-8.

102. Pickles T. Prostate-specific antigen (PSA) bounce and other fluctuations: Which biochemical relapse definition is least prone to PSA false calles? An analysis of 2030 men treated for prostate cancer with external beam or brachytherapy with or without adjuvant androgen deprivation therapy. Int J Radiat Oncol Biol Phys 2006;64:1355-59.

103. Mazeron R, Bajard A, Montbarbon X, Gassa F, Malet C, Rocher F, Clippe S, Bringeon G, Desmettre O, Pommier P. Permanent 125I-seed prostate brachytherapy: early prostate specific antigen value as a predictor of PSA bounce occurrence. Radiat Oncol 2012 Mar 26;7:46

104. Ciezki JP, Reddy CA, Garcia J, Angermeier K, Ulchaker J, Mahadevan A, Chehade N, Altman A, Klein EA. PSA kinetics after prostate brachytherapy: PSA bounce phenomenon and its implications for PSA doubling time. Inj J Radiat Oncol Biol Phys. 200662 (2):512-7.

105. Grob BM, Schellhammer PF, Brassil DN, Wright GL Jr. Changes in immunohistochemical staining of PSA, PAP, and TURP-27 following irradiation therapy for clinically localized prostate cancer. Urol 1994:44:525-9.

106. Hussain M, Goldman B, Tangen C, et al. Prostate-specific antigen progression predicts overall survival in patients with metastatic prostate cancer: data from Southwest Oncology Group trials 9346 (Intergroup Study 0162) and 9916. J Clin Oncol 2009; 27(15):2450-6.

107. Scher HI, Halabi S, Tannock I, et al. Design and end points of clinical trials testosterone: recommendations of the postate cancer clinical trials working group. J Clin Oncol. 2008 Mar 1; 26(7):1148-59.

108. Qin J, Liu X, Laffin B, et al. The PSA (-/lo) prostate cancer cell population harbors self-renewing long-term tumor-propogating cells that resist castration. Cell Stem Cell 2012; 10(5):556-69.

109. Bubendorf L, Schopfer A, Wagner U, et al. Metastatic Patterns of Prostate Cancer: an Autopsy Study of 1589 Patients. Hum Pathol. 2000;31:578-583.

110. Messiou C, Cook G, de Souza NM. Imaging metastatic bone disease from carcinoma of the prostate. Br J Cancer 2009;101:1225-1232.

111. Pollen JJ, Witztum KS, Ashburn WL. The flare phenomenon on radionuclide bone scan in metastatic prostate cancer. Am J Roentgenol 1984;142:773-776.

112. Weber G. Enzymology of cancer cells. N Engl J Med 1977;296:541-551.

113. Bouchelouche K, Oher P. Positron emission tomography/computed tomography of urological malignancies: an update review. J Urol 2008; 179:34-45.

114. Pinto F, Totaro A, Palermo G, et al. Imaging in prostate cancer staging: present role and future perspectives. Urol Int 2012;88:125-36.

115. Ludwig JA, Weinstein JN. Biomarkers in cancer staging, prognosis and treatment selection. Nat Rev Cancer 2005;5:845-56.

116. Bussemakers MJ, van Bokhoven A, Verhaegh GW, et al. DD3: a new prostate-specific gene, highly overexpressed in prostate cancer. Cancer Res. 1999;59(23): 5975-9.

117. Neves AF, Araújo TG, Biase WK, et al. Combined analysis of multiple mRNA markers by RT-PCR assay for prostate cancer diagnosis. Clin. Biochem. 2008;41(14-15): 1191-8.
118. Neves AF, Araújo TG, Biase WK, et al. Combined analysis of multiple mRNA markers by RT-PCR assay for prostate cancer diagnosis. Clin. Biochem. 2008;41(14-15): 1191-8

119. Ploussard G, Durand X, Xylinas E, Moutereau S, et al. Prostate cancer antigen 3 score accurately predicts tumour volume and might help in selecting prostate cancer patients for active surveillance. Eur Urol 2011;59(3):422-9.

120. Hayes JD, Pulford DJ. The glutathione S-transferase sugergene family: regulation of GST and the contribution of the isoenzymes to cancer chemoprotection and drug resistance. Crit Rev Biochem Mol Biol 1995;30:445-600.

121. Bastian PJ, Ellinger J, Schmidt D, et al. GSTP1 hypermethylation as a molecular marker in the diagnosis of prostatic cancer: is there a correlation with clinical stage, gleason grade, PSA value or age? Eur J Med Res 2004;9:2673-7.

122. Murphy GP, Kenny GM, Ragde H. Measurement of serum prostate-specific membrane antigen, a new prognostic marker for prostate cancer. Urology 1998;51(suppl 5a):89-97.

123. Israeli RS, Powell CT, Corr JG et al. Expression of the prostate specific membrane antigen. Cancer Res 1994;54(7):1807-11.

124. Brawer MK, Chetner MP, Beatie J, et al. Screening for prostatic carcinoma with prostate specific antigen. J Urol $1992 \mathrm{Mar} ; 147(3 \mathrm{Pt} 2): 841-5$.

125. Sreekumar A, Poisson LM, Rajendiran TM, et al. Metabolomic profiles delineate potential role for sarcosine in prostate cancer progression. Nature 2009; 457:799-800.

126. Couzin J. Metabolite in urine may point to high-risk prostate cancer. Science 2009;323:865.

127. Johansson JE, Andrén O, Andersson SO, Dickman PW, Holmberg L, Magnuson A, Adami HO. Natural history of early, localized prostate cancer. JAMA. 2004, 291:2713-9.

128. Etzioni R, Penson DF, Legler JM, di Tommaso D, Boer R, Gann PH, Feuer EJ. Overdiagnosis due to prostate-specific antigen screening: lessons from U.S. prostate cancer incidence trends. J Natl Cancer Inst. 2002, 94:981-90.

129. Tsodikov A, Szabo A, Wegelin J. A population model of prostate cancer incidence. Stat Med. 2006, 25:2846-66.

130. Kirkham AP, Emberton M, Allen C: How good is MRI at detecting and characterising cancer within the prostate? Eur Urol. 2006, 50:1163-74.

131. Wefer AE, Hricak H, Vigneron DB, Coakley FV, Lu Y, Wefer J, Mueller-Lisse U, Carroll PR, Kurhanewicz J. Sextant localization of prostate cancer: comparison of sextant biopsy, magnetic resonance imaging and magnetic resonance spectroscopic imaging with step section histology. I Urol. 2000, 164:400-4

132. Scheidler J, Hricak H, Vigneron DB, Yu KK, Sokolov DL, Huang LR, et al. Prostate cancer: localization with three-dimensional proton MR spectroscopic imaging-clinicopathologic study. Radiology 1999; 213: 473-80.

133. Haider MA, Kwast TH, Tanguay J, et al. Combined T2-weighted and diffusion-weighted MRI for localization of prostate cancer. AJR Am J Roentgenol. 2007;189:323-328.

134. Lim HK, Kim JK, Kim KA, Cho KS. Prostate cancer: apparent diffusion coefficient map with T2-weighted images for detection - a multireader study. Radiology. 2009;250:145-151.

135. Hara N, Okuizumi M, Koike H, Kawaguchi M, Bilim V. Dynamic contrast-enhanced magnetic resonance imaging (DCE-MRI) is a useful modality for the precise detection and staging of early prostate cancer. Prostate. 2005;62:140-147.

136. Alonzi R, Padhani AR, Allen C. Dynamic contrast enhanced MRI in prostate cancer. Eur J Radiol. 2007;63:335-350.

137. Chen M, Dang HD, Wang C, et al. Prostate Cancer Detection: Comparison of T2-Weighted Imaging, Diffusion-Weighted Imaging, Proton Magnetic Resonance Spectroscopy Imaging, and the Three Techniques Combined. Acta Radiologica. 2008;5:602-610.

138. Vargas HA, Akin O, Franiel T et al: Diffusion-weighted endorectal MR imaging at $3 \mathrm{~T}$ for prostate cancer: tumor detection and assessment of aggressiveness. Radiology. 2011;259:775.

139. deSouza NM, Riches SF, Vanas NJ et al. Diffusion-weighted magnetic resonance imaging: a potential non-invasive marker of tumour aggressiveness in localized prostate cancer. Clin Radiol. 2008;63:774.

140. Margel D, Yap SA, Lawrentschuk N et al. Impact of multiparametric endorectal coil prostate magnetic resonance imaging on disease reclassification among active surveillance candidates: a prospective cohort study. J Urol. 2012;187:1247.

141. Washington SL, Bonham M, Whitson JM, Cowan JE, Carroll PR. Transrectal ultrasonography-guided biopsy does not reliably identify dominant cancer location in men with low-risk prostate cancer. BJU Int. 2012;110(1):50-5.

142. Wilson DH, Hanlon DW, Provuncher GK, Change L, et al. Fifth-generation digital immunoassay for prostate specific antigen using single molecule arrays. Clin Chem. 2011;57(12): 1712-1721.

143. Locke JA, Zafarana G, Ishkanian AS, et al. NKX3.1 haploinsufficiency is prognostic for prostate cancer relapse following surgery or image-guided radiotherapy. Clin Cancer Res 2012;18:308-16.

144. Nuzzo PV, Rubagotti A, Zinoli L, Ricci F, Salvi S, Boccardo S, Boccardo F. Prognostic value of stromal and epithelial periostin expression in human prostate cancer: correlation with clinical pathological features and the risk of biochemical relapse or death. BMC Cancer 2012, 12:625.

145. Freedland SJ, Presti JC Jr, Amling CL. Time trends in biochemical recurrence after radical prostatectomy. Urology 2003; 61:736-741 
146. Han M, Partin AW, Zahurak M, Piantadosi S, Epstein JI, Walsh PC. Biochemical (prostate specific antigen) recurrence probability following radical prostatectomy for clinically localized prostate cancer. J Urol 2003;169:517-523

147. Panebianco V, Barchetti F, Sciarra A, et al. Prostate cancer recurrence after radical prostatectomy: the role of 3-T diffusion imaging in multi-parametric magnetic resonance imaging. Eur Radiol 2013; 23:1745-1752

148. Cirillo S, Petracchini M, Scotti L et al. Endorectal magnetic resonance imaging at 1.5 Tesla to assess local recurrence following radical prostatectomy using T2-weighted and contrast-enhanced imaging. Eur Radiol 2009;19:761-769

149. Dearnaley DP, Sydes MR, Graham JD, et al. Escalated-dose versus standard-dose conformal radiotherapy in prostate cancer: first results from the MRC RT01 randomised controlled trial. Lancet Oncol 2007; 8:475-487

150. Tamada $\mathrm{T}$, Sone $\mathrm{T}$, Jo $\mathrm{Y}$, et al. Locally recurrent prostate cancer after high-dose-rate brachytherapy: the value of diffusion-weighted imaging, dynamic contrast-enhanced MRI, and T2-weighted imaging in localizing tumors. Am J Roentgenol. 2011;197: 408-14.

151. Westphalen AC, Reed GD, Vinh PP, et al. Multiparametric 3T endorectal MRI after externalbeam radiation therapy for prostate cancer. J Magn Reson Imaging. 2012;36:430-7.

152. Coakley FV, Teh HS, Qayyum A, et al. Endorectal MR imaging and MR spectroscopic imaging for locally recurrent prostate cancer after external beam radiation therapy: preliminary experience. Radiology 2004; 233:441-448

153. Nunez R, Macapinloc HA, Yeung HW, et al. Combined 18F-FDG and 11C-methionine PET scans in patients with newly progressive metastatic prostate cancer. J Nucl Med 2002;43:46-55.

154. Brogsitter C, Zophel K, Kotzerke J. (18)F-choline, (11)C-choline and (11)C-acetate PET/CT: comparative analysis for imaging prostate cancer patients. Eur J Nucl Med Mol Imaging 2013; 40(suppl 1):18-27.

155. Watahiki A, Macfarlane RJ, Gleave ME, et al. Plasma miRNAs as biomarkers to identify patients with castration-resistant metastatic prostate cancer. In J Mol Sci 2013;14(4):7757-70.

156. Yoon HY, Kim YW, Kang HW, et al. Pyrosequencing analysis of APC methylation level in human prostate tissues: a molecular marker for prostate cancer. Korean J Urol. 2013 Mar;54(3):194-8.

157.Som A, Tu SM, Liu J, et al. Response in bone turnover markers during therapy predicts overall survival in patients with metastatic prostate cancer: analysis of three clinical trials. Br J Cancer 2012;107(9):1547-53. 